\title{
Broad-Spectrum Antibiotic Abuse and its Connection to Obesity.
}

\author{
Brent Gawey and Krzysztof Czaja* \\ Department of Veterinary Biosciences \& Diagnostic Imaging, College of Veterinary Medicine, The University of Georgia, Athens, \\ USA.
}

Received: April 13, 2017; Accepted: May 05, 2017; Published:June 01, 2017

*Corresponding author: Krzysztof Czaja, DVM, Ph.D., Department of Veterinary Biosciences \& Diagnostic Imaging, The University of Georgia College of Veterinary Medicine, 501 D.W. Brooks Drive, Athens, GA 30602, United States, E-mail: czajak@uga.edu

\begin{abstract}
The purpose of this review was to assess patterns of antibiotic prescriptions across the United States and evaluate their relationship with associated conditions. A focus on national antibiotic prescriptions and proposed side effects was studied from 1993-2008. State-to-state differences in antibiotic prescribing and associated patterns of obesity were studied from 2011-2014. Data for these study periods was extracted from the CDC and affiliated institutions. Correlation assessments between antibiotic prescription patterns and a set of variables related to the proposed mechanism of antibiotic effects were made. From 2011-2014, cephalosporin prescriptions demonstrated the strongest relationship with associated obesity rates, while beta-lactams (cephalosporins and penicillins) showed weaker relationships when taken as a holistic class. To make light of these results, drug use of Ceftriaxone, a broad-spectrum, third-generation cephalosporin and Amoxicillin, a narrow-spectrum penicillin, was compared from 1993 to 2008 in relation to the prevalence of various health conditions: obesity; diabetes mellitus; sleep disorders; endocrine, nutritional, metabolic diseases and immunity disorders; and infectious and parasitic diseases. Ceftriaxone demonstrated a strong, significant, and positive relationship with all of the above variables, except for parasitic and infectious diseases. By comparison, Amoxicillin showed weak and non-significant relationships with all of the above variables. Despite their correlational limitations, these results suggest that broad-spectrum antibiotics play a significant role in the development and persistence of many chronic conditions in the United States. A mechanism providing a hypothesized explanation for these results was proposed based on extensive literature review.
\end{abstract}

\section{List of Abbreviations}

3gc: Third-generation cephalosporins; BRFSS: Behavioral Risk Factor Surveillance System; CDC: Center for Disease Control and Prevention; CRH: Corticotrophin-releasing hormone; GI: Gastrointestinal; HPA axis: Hypothalamic-pituitaryadrenal axis; NAMCS: National Ambulatory Medical Care Survey; SCFA: Short-Chain Fatty Acids

\section{CHAPTER I}

\section{Introduction}

\section{Obesity \\ Determination of Obesity}

An individual's body composition can be computed through a variety of different methods. Perhaps the most widespread measure involves the determination of a person's Body Mass Index (BMI). BMI is a formula that outputs a number based on the ratio of a person's weight in kilograms to the square of their height in meters. The classification of a person's BMI is divided into four categories: underweight $(<18.50)$, normal range (18.50-24.99), overweight ( $\geq 25.00$ ), and obese ( $\geq 30.00$ ) [1]. Past and present statistics regarding the weight status of Americans have been calculated through self-report of height and weight through telephone communications as an effort of the CDC's Behavioral Risk Factor Surveillance System (BRFSS). However, starting in 2010, the BRFSS switched from landline telephone contact to cell phone communicated data to include a larger and more accurate representation of self-reported health information. For this reason, any collection of obesity data from 2011 to the present cannot be compared to data prior to 2010 [2]. BMI serves as a baseline measurement of an individual's weight classification, however there are certain clinical limitations of BMI that should be noted. First, BMI does not distinguish between muscle mass and fat mass in the measurement of a person's weight. As a result, an individual with large amounts of muscle mass and minimum fat might be classified as obese despite their relatively positive state of health, and an individual with hidden, visceral fat and metabolic syndrome could be classified in the normal range for BMI, leading to misinterpretations of the measure. Secondly, BMI does not distinguish between waist circumferences, which could confound the output of the measure based on an individual's body shape. However, despite these limitations, BMI is currently the most effective way to sample a large amount of individuals nationwide to gather a rough estimate of the weight classification of a majority of Americans. 
More accurate measures of body composition exist that would be more ideal in the calculation of national weight characteristics. Dual-energy X-ray Absorptiometry (DXA) allows for a determination of intramuscular fat and bone density to present a more accurate understanding of a person's body composition than what is allowed through BMI. Additionally, Hydrostatic Weighing allows for a measurement of bone, muscle, fat, and water density of a person. Body fat is calculated through a comparison with the overall body density. While these methods are more accurate in determining an individual's body composition, limitations such as cost and availability constrict their use on a national scale of comparison.

\section{Obesity has become pandemic.}

Since 1980, the worldwide proliferation of obesity has more than doubled, and despite the preventable nature of obesity, obesity-related mortality grossly outnumbers mortality stemming from complications of being underweight [3]. Over time, obesity has typically been stigmatized as a problem unique to high-income countries, however its prevalence has begun to become more common in low-to middle-income developing countries. For example, the number of obese children in Africa has doubled from 1990 to 2014, with an estimated 10.6 million obese children [4]. The root cause of these increases is generally unknown at this point, however a combination of dietary, physical, and pharmaceutical changes are believed to play a synergistic role in the current patterns being observed on a global scale for obesity.

In the United States, obesity is classified as an epidemic, and while most epidemics are controlled over time, obesity rates have continued to rise in dramatic fashion. From 1950 to 2014, obesity rates have more than tripled from $10 \%$ to $38 \%$ nationwide in U.S. adults. In fact, currently, one in three US adults are obese. However, this trend in obesity rates is not limited to adults, as childhood (ages 2 to 19) obesity rates have tripled from 1980 to 2014, placing children at a greater risk for developing chronic diseases earlier in life. To further highlight the dramatic increase in obesity prevalence across the United States, it is important to note that in 1990 no state had a prevalence of obesity greater than $15 \%$, whereas in 2015 , no state had an obesity prevalence lower than $20 \%$ [5]. Currently, the South has the highest prevalence of obesity in the United States at an average of $31.2 \%$, trailed by the Midwest (30.7\%), the Northeast (26.4\%), and finally the West (25.2\%). In particular, Alabama, Louisiana, Mississippi, and West Virginia had the greatest prevalence of obesity in 2015 with rates equal to and greater than $35 \%$ [6].

\section{Obesity presents major threats to healthcare costs.}

Living in an obese state predisposes an individual to a variety of life-threatening conditions. For example, obese people have been found to more frequently develop cardiovascular diseases (heart disease and stroke), diabetes, hypertension, allergies, chronic disease, musculoskeletal disorders (arthritis), and cancer (breast, ovarian, pancreatic, colon, kidney, thyroid, gallbladder, and esophageal) than individuals maintaining a healthy body composition $[7,8]$. It is therefore unsurprising that both the incidence and prevalence of chronic, inflammatory diseases have increased over time, with an estimated $45 \%$ of the American population currently suffering from a chronic disease [9]. The propensity of chronic disease development from obesity leads to enormous costs for the US healthcare system. In one year, obesity-related health care costs total more than $\$ 147$ billion dollars in preventable healthcare spending. This amount makes up nearly $21 \%$ of annual medical spending. Even more staggering, childhood obesity alone is responsible for $\$ 14$ billion in direct medical costs each year. It is predicted that if obesity continues to increase, obesity-related health care costs could rise to anywhere from $\$ 195$ to $\$ 213$ billion by 2030 [10]. In addition, current estimates predict 500,000 additional cases of cancer in the United States by 2030 if obesity continues to follow current trends [11].

\section{Antibiotics}

\section{History of Antibiotics}

In the past, infectious disease was a normal part of life. In $1900,30.4 \%$ of all deaths occurred in children less than the age of 5 . The leading causes of death were pneumonia, tuberculosis, and diarrhea, accounting for one third of all deaths [12 a]. From 1918-1919, more people died from an influenza pandemic than collectively in combat in World War I, World War II, the Korean War, and the Vietnam War [12 b]. However, in 1928, medicine was revolutionized. Bacteriology professor, Sir Alexander Fleming, discovered the first true antibiotic, penicillin. Since their first use to treat infections in the 1940s, antibiotics have become one of the most impactful discoveries in medicine, attributable for the saving of millions of lives on a global scale. In 1945, just around the time World War II was ending, the average life expectancy staggered around 50 years. However, by 1990, with the widespread use of antibiotics to treat infectious diseases, the average life expectancy rose to around 70 years [13]. The number of deaths in children under five sunk from $30.4 \%$ in 1900 to $1.4 \%$ in 1997 [12]. But with all good things, overuse can lead to detriment. Currently, almost every antibiotic ever developed is subject to bacterial resistance, rendering the effect of the drugs useless, and potentially harmful, making infectious disease a potential threat to society once again [14]. For example, nearly 2 million people in the United States are infected with antibiotic-resistant bacteria, and almost 23,000 perish due to these infections [15]. Based on the level of increased antibiotic resistance, a new wave of "next-generation" antibiotics are being created, with modified side chain structures to introduce new properties and potentially restore the effectiveness of certain classes of antibiotics, however, due to the incredibly adaptive nature of bacterial populations, perhaps the modern age of antibiotics as we know it is coming to an end.

\section{Mechanism of Antibiotic Action}

Antibiotics differ in their tissue distribution, rate of elimination, and effects in a living organism. However, the main distinction between antibiotics lies primarily in their differing mechanisms of action. Examples include inhibition of cell wall synthesis, inhibition of protein synthesis, inhibition of nucleic 
acid synthesis, inhibition of metabolic pathways, and interference with cell membrane integrity. Classification of antibiotics by mechanism along with examples of common drugs in each class is shown in in Table 1 (Appendix). preceding the study. Participants were not specifically endurance trained but physically active sport students. Anthropomorphic characteristics of the participants are indicated in Table 1.

\begin{tabular}{|c|c|}
\hline Table 1: Classification of antibiotics by mechanism [16] \\
\hline $\begin{array}{c}\text { Targeted inhibition/ } \\
\text { interference }\end{array}$ & Examples of Antibiotics \\
\hline Cell Wall Synthesis & $\begin{array}{c}\text { B-lactam drugs (Penicillins, } \\
\text { Cephalosporins) }\end{array}$ \\
\hline & Vancomycin \\
\hline Protein Synthesis & Bacitracin \\
\hline & Chloramphenicol \\
\hline & Tetracyclines \\
\hline & Macrolides \\
\hline Cell Membrane & Lincosamides \\
\hline & Polymxin B \\
\hline Nucleic Acids & Amphotericin \\
\hline & Fluoroquinolones (Ciproflaxin) \\
\hline Metabolic Pathways & Rifamycin \\
\hline
\end{tabular}

\section{Antibiotics are classified by spectrum of activity}

Antibiotics are classified as broad or narrow-spectrum based on the range of bacterial species susceptible to their action. Broad-spectrum antibiotics act against both Gram-positive and Gram-negative bacteria, while narrow-spectrum antibiotics are only effective against either Gram-positive or Gram-negative bacteria. Narrow-spectrum antibiotics are generally limited in use due to the requirement of pathogen class identification before beginning treatment. In comparison, broad-spectrum antibiotics can be employed regardless of whether or not the infective bacterial species has been identified, and are often used in cases when the prescriber is not sure the exact pathogenic nature of the infection [17]. Therefore, based on the spectrum of activity, antibiotics differ in their altering effects of the composition of bacterial populations, with broad-spectrum antibiotics having a greater impact on bacterial flora contained throughout the body. Common examples of broad-spectrum antibiotics include azithromycin, amoxicillin, tetracycline, and quinolones. The most common narrow-spectrum antibiotics include glycopeptides and bacitracin for treatment of Gram-positive infections and polymixins for the treatment of Gram-negative bacterial infections [18].Currently, broad-spectrum antibiotics are the most commonly prescribed class of antibiotic, with amoxicillin prescribed the most for children and teens and azithromycin the most prescribed for older adults [18].

\section{Broad-spectrum antibiotic use has increased over time.}

In 1995, a total of 75 million antibiotic prescriptions were written for adults in the outpatient setting in the United States. This number increased to 95 million in 2002, and based on the latest data collected by the Center for Disease Control, the number of antibiotic prescriptions written in the outpatient setting climbed to a staggering 262.5 million from 2013 to 2014 [19]. This increase alone is something to marvel at, as more than 5 out of every 6 Americans takes an antibiotic every year. However, the more concerning area of increase in antibiotic prescription rates involve the changes in the type of antibiotics being prescribed. In 1950, 7,479 narrow-spectrum antibiotics and 0 broad-spectrum antibiotics were prescribed in the United States. In 2002, the number of narrow-spectrum antibiotics prescribed increased to 63,428 and broad-spectrum antibiotic prescriptions to 13 million $[20,21]$. This increase in broad-spectrum antibiotics has not slowed, as broad-spectrum antibiotic prescriptions have doubled from 2000 to 2010 [22]. In addition, it was recently discovered that at least 1 in 3 antibiotic prescriptions in the United States are unnecessary, and antibiotics are often deployed to treat viral infections [23].

During his speech upon the reception of the Nobel Peace Prize in 1945, Sir Alexander Fleming marveled at the potential power of antibiotics in medicine. However, in a later interview with The New York Times, Fleming warned about the consequences of misuse. Sir Fleming proclaimed, "The thoughtless person playing with penicillin treatment is morally responsible for the death of the man who succumbs to infection with the penicillin-resistant organism." He followed this by warning, "Public will demand penicillin and ... then will begin an era ... of abuses [24]. Clearly, this era of abuse is underway.

\section{Gut Health \\ Humans are covered with microbes}

Recent estimates suggest that the normal flora of a single individual contains anywhere from 40 to 100 trillion microbial cells. This microbial colonization begins at birth and varies in progression and composition based on a variety of factors such as diet, drug exposure, contact with other people, travel, and respiration. These microbial floras are harbored all over the human body, including the skin, in the oral cavity and sinuses, the esophagus, digestive tract, lower urogenital tract, and in the external ear canal and eye. In terms of species diversity, the highest levels exist in the gut and oral flora, whereas the urogenital tract has the lowest amount of bacterial diversity.

The human microbiome is composed of four main groups of bacteria. These include Firmicutes, Bacteroidetes, Proteobacteria and Actinobacteria, with Firmicutes and Bacteroidetes accounting for more than $80 \%$ of the microbiota in our gut. Firmicutes consists of mostly Gram-positive bacteria, with low amounts of Gram-negative bacteria. Bacteroidetes consists solely of Gram-negative bacteria, with primary representation by the Bacteroidetes genus in the human gut. Proteobacteria consist of Gram-negative bacteria and include many pathogenic bacteria. Actinobacteria are primarily Gram-positive bacteria involved in 
metabolite production [25].

Recent progress of the Human Microbiome Project has produced the estimate that the number of microbial genes (bacteria, fungi, protozoa, and viruses) contained in one person is 200 times greater than that of the human genome [26]. The sum of all of this microbial genome contained in and on the human body is collectively termed the microbiome. Therefore humans can be viewed as "super organisms", with two genomes: one that is inherited from our parents and a much more dynamic one that is shaped over the course of our life. However, despite the relative abundance of microbes and microbial genetic material harbored throughout the human body, the majority of our microbial flora is contained in the gut, particularly the large intestine. Therefore the gut microbiota are an area of particular importance in regards to alterations induced to our normal flora as a result of human behavior and drug intake [27].

The bacteria harbored inside of the human body are commonly referred to as the "hidden organ" due to the often overlooked, yet critical role they play in human physiology, metabolism, immune system regulation, development, protection against other disease causing bacteria, elimination of chemical toxins, production of vitamins and amino acids, and mental health [28, 29]. Therefore, the maintenance of gut health is crucial for the maintenance of good health, and any changes to our normal flora can have significant consequences to our health for better or for worse.

The composition of bacteria harbored throughout an individual's body is unique and can be rapidly altered by a variety of factors including, but not limited to diet, lifestyle, and exposure to toxins and antibiotics. In recent years, it has been discovered that microbiome composition is a key environmental factor that drives human genetic expression, with the power to turn genes on and off based on the ratios of microbes present [30]. In this regard, there are no good or bad bacteria when they are maintained in normal ratios. However, an individual's microbial makeup can become harmful if certain bacterial species are allowed to outnumber other beneficial strains of bacteria. For example, studies have shown that autistic children have a quantitatively and qualitatively different composition of bacteria in their intestinal flora in comparison to healthy children [31, 32]. Additionally, autoimmune diseases such as diabetes, rheumatoid arthritis, muscular dystrophy, and multiple sclerosis have been found to be associated with dysbiosis of the intestinal flora [33]. To further highlight the importance of the maintenance of the gut flora, experiments comparing germ free mice to conventional mice have found nutritional deficiencies, decreased epithelial cell renewal, decreased lymphoid tissue, cecum distension, and increased susceptibility to pathogenic infection in germ free mice when compared to conventional mice [34].

\section{Gut bacteria play a crucial role in human health.}

The gut microbiome hosts over 1000 bacteria species with an accompanying 5 million bacterial genes that play an important role in human digestion [35]. Due to the lack of digestive enzyme production by the epithelial cells of the mammalian large intestine, the function of the resident microbial communities is paramount. These colonizing bacteria in the human large intestine encode a wide variety of digestive enzymes that can elicit a wide variety diversity of metabolic capabilities in comparison to the human host [35]. The primary pathway through which bacteria accomplish their metabolic function is fermentation. Fermentation is the enzymatic decomposition and utilization of substrates, such as dietary carbohydrates and glycans that are unable to be enzymatically digested in the small intestine [36]. The end products of fermentation are short chain fatty acids, lactic acid, methane, hydrogen, and carbon dioxide [37 a]. Therefore the next time an individual experiencing an increased rate of flatulence is encountered, don't blame them, blame their bacteria. However, perhaps the most important byproducts of bacterial fermentation from a metabolic standpoint are the short chain fatty acids, which include acetic, propionic, and butyric acids, with butyric acid being the preferred energy source for bacteria inhabiting the colon. These weak, short chain fatty acids lower the colonic $\mathrm{pH}$, which has a direct on the microbial composition of the gut [37 b]. Additionally, these fatty acids can be readily metabolized within human epithelial cells of the large intestine and distributed as a source of energy throughout the body due to their hydrophobic nature.

Additionally, bacteria are an important source of vitamin K2 and B synthesis. Vitamin K2 plays an essential role in human health as it is used to activate proteins involved in the regulation of calcium deposits in the body [38]. This is important to human health, as it has been found that low levels of vitamin $\mathrm{K} 2$ can lead to increased levels of arterial calcification, predisposing an individual to heart disease [39]. B vitamins produced in the gut (Folate, Biotin, Vitamin B12, Thiamine, and Niacin) also play a critical role in human health with effects ranging from energy extraction from food to DNA and hormone synthesis [40].

\section{The mucosal lining of the gut is tied to immune regula- tion.}

The gut mucosa is the innermost lining of the gastrointestinal tract and is properly a mucous membrane that lines the digestive tract, serving a functional role in nutrient absorption and as a barrier between the gut and the rest of the body [40]. This inner lining of the intestinal tract is composed of a layer of epithelial cells, connective tissue, and a layer of smooth muscle.

The only three places in the body that contain mucosal linings are the respiratory, urogenital, and intestinal tract. All of these tracts are associated with bacteria and serve a similar function, keep bacteria where they should be. The gut mucosa therefore has an important role as it serves as the interface between the host and the "outside" microbial world. Therefore, a healthy mucosal lining serves as a double-edged sword, supporting the growth of commensal microbes, inhibiting the growth of pathogenic bacteria, and allowing for the exchange of nutrients and metabolic products [41]. However, only a limited number of bacterial species can "access" the mucosal lining. These three gram-positive, commensal bacteria colonizing the mucosal lining are Clostridium, Lactobacillus, and Enterococcus [42]. These mucosal bacteria play a critical role in the selective permeability and regulation of the mucosal layer of the intestinal epithelium 
by contributing and localizing tight junction proteins at epithelial cell boundaries [43].

In addition to harboring commensal bacteria, the mucosal membrane of the intestinal epithelium plays an important role in the moderation of immune responses in the host, primarily through utilization of Immunoglobulin A and Toll-like receptors [44]. In particular, Secretory IgA (SIgA) serves as the first line of immune defense against enteric toxins and pathogenic microbes in the intestinal epithelium, known as immune exclusion. SIgA is contained in the mucosal lining, which traps antigens and pathogenic microbes and ensures their proper clearance from the intestinal lumen before they can reach epithelial receptors. In addition, SIgA has been recently discovered to destroy bacterial virulence factors, directly influence the composition of the intestinal microbes via signaling pathways, and down regulate the pro-inflammatory response normally resulting from contact with pathogenic bacteria and food antigens [45].

Bacteria play an important role in the development and maintenance of the mucosal lining and mucosal immune system. This role is particularly emphasized in childhood development, as the proper development of a newborn child's mucosal lining is directly influenced by gut microbes [46]. In an experiment using gnotobiotic mice, it was shown that colonization of mucosal surfaces is highly protected, allowing the selective development of only commensal bacteria that in turn stimulate the development of the postnatal systemic immune response. However, these systemic immune responses are later shifted to a state of tolerance once the Toll-like receptors begin to recognize the pattern of microbes commensally inhabiting the mucosal lining, allowing for a unique mucosal immunity [47]. It is well documented that disruptions to the mucosal lining cascades to impaired mucosal barrier function and immune regulation, leading to increased penetration of pathogenic bacteria and food antigens into systemic circulation. This "leakage" elicits an exaggerated general immune response, often manifested symptomatically as allergies, systemic inflammation, irritable bowel disease, autoimmune diseases, rheumatoid arthritis, and diabetes [44].

When intestinal permeability reaches a chronic level, leaky gut syndrome or "intestinal hyper-permeability", can develop as a result of tight junction malfunction and errant signaling of the permeability-regulating protein, zonulin [48]. The translocation of gut microbiome derived endotoxins, in particular Lipo polysaccharide (LPS), as a result of this increased intestinal permeability has been properly termed Metabolic Endotoxemia (ME) [49]. In contrast, healthy individuals have low endotoxins in circulation, whereas individuals prone to weight gain and atherosclerosis contain elevated levels of endotoxins in their systemic circulation50. It is believed that the increased permeability of the intestinal lining of the mucosal layer of the gut leads to the activation of toll-like receptor-mediated immune activation that elicits a state of chronic, systemic, low-grade inflammation. In other, related studies, it was found that weight gain and the subsequent progression towards obesity can be characteristically identified through an increased intestinal permeability, which allows for increased translocation of LPS into the blood stream, that elicits in a two-to-threefold increase in blood serum concentrations. This concentration of endotoxins in the bloodstream is at the threshold level to be properly classified as metabolic endotoxemia. Serum levels of this magnitude are causative for inflammatory activation and oxidative stress, two conditions commonly found in obese individuals [51]. In another study investigating plasma endotoxin (LPS) levels in the bloodstream, it was discovered that a high-fat meal promotes the absorption of LPS across the intestinal barrier, leading to plasma levels characteristic of ME [50]. Previous studies also support these findings, demonstrating that meals high in fatty acids were linked with increased intestinal barrier permeability $[52,53]$. Further investigation elevated endotoxin levels in the bloodstream found that merely $10 \mathrm{pg}$ endotoxin $/ \mathrm{mL}$ caused a significant increase in endothelial cell expression of Eselectin for 6 hours [50]. E-selectin is a cell adhesion molecule expressed by inflamed endothelial cells in response to cytokine activation, which elicits a downstream chronic, acute inflammatory response, demonstrating the immune activity LPS presence in the bloodstream produces. The results of these experiments clearly show how important a healthy mucosal lining is towards the maintenance of good health, as even minor disruptions to the mucosal lining and intestinal epithelium allow for the translocation of LPS into the bloodstream, which causes a cascade of lowgrade, systemic inflammation.

\section{Gut composition differs between obese and lean indi- viduals.}

Obesity is a complex disease, developing primarily from unbalanced energy intake and expenditure over an extended period of time. In terms of our microbiota, obesity has been linked to characteristic phylum-level changes in the functional diversity of bacteria, with obese people demonstrating lower levels of overall gut flora diversity in comparison to lean individuals [54]. However, the distinction here lies in the fact that obese people don't necessarily contain less bacteria, rather they contain a lower diversity of gut flora on a phylum level. A low level of gut diversity is problematic because each strain of bacteria holds a unique function involved in the digestion and metabolism of nutrients. Therefore, a decreased diversity of bacteria leads to a decreased functionality of the intestinal flora, which can have severe implications for an individual's health. The decrease in diversity is specifically associated with an increased abundance of Firmicutes bacteria, with a lower level of Bacteroidetes microbiota [55]. Another study discovered that the gut microbiome of an obese twin was less diverse and contained a higher level of enzymes than the lean twin. The higher level of enzymes indicates a higher level of food digestion and calorie absorption from food in the obese twin, which is believed to be associated with the increased weight of the obese twin [57].

Further research has shown that elimination of certain species of microbes can trigger obesity. In one study, it was found that elimination of Lactobacillus, Allobaculum, Rikenellece$a e$, and Candidatus arthromitus led to metabolic changes in rats that eventually welcomed the onset of obesity [56]. To further highlight the impact the composition of an individual's intestinal flora can have on the tendency to gain weight, it was discovered that when germ free mice were given fecal transplants from an 
obese or lean individual, they adapted the metabolic and physical tendencies of their respective donor, with "fat mouse bacteria" causing the mouse to gain weight and "lean mouse bacteria" causing the mouse to maintain a lean mass despite both being fed the same diet [58]. In yet another study, it was discovered that a group of digestion-improving microbes were found almost exclusively in obese individuals and are found in trace amounts in normal weight individuals, further highlighting the metabolic consequences gut flora composition can have on its host [59]. Implications of these floral shifts were further investigated in a study that found lean people contained larger quantities of beneficial bacteria whereas people with excess weight were found to have greater quantities of pathogenic bacteria. This presence of pathogenic bacteria is believed to result in an inflammatory response throughout the body through an interaction with fat cells, which can lead to leptin resistance and the development of diabetes and chronic disease [60].

Despite the findings above, the gut exists as a relatively dynamic organ. One study found that the disrupted ratio of Bacteroidetes and Firmicutes in obese individuals was restored to its normal balance when the individual began to lose weight through the maintenance of a low-calorie diet [61]. One study attempted to explain these observations by concluding that the obese microbiome has an increased capacity to harvest energy from the diet. Additionally, this study highlighted the microbiome as a causal factor in the development of obesity by demonstrating that the colonization of germ free mice with obese microbiota led to increased amounts of body fat in comparison to germ free mice colonized with lean microbiota [62]. Another study further investigating the connection of the microbiome to obesity found that infusion of endotoxin-producing Enterobacter from the gut of obese humans into germ-free mice, induced obesity and insulin resistance through an inflammation-mediated pathway. However, when the abundance of Enterobacter was found to decrease from $35 \%$ to non-detectable, the mouse was also observed to lose $51.4 \mathrm{~kg}$ of its original $174.8 \mathrm{~kg}$ mass and recover from hyperglycemia and hypertension after only a 23 -week dietary adjustment period. Additionally, this study found that it was not possible to induce obesity in gnotobiotic mice simply through the introduction of a diet high in fat if the Enterobacter strain was not introduced first. However, when Enterobacter was introduced before the high fat diet was started, the mice gained weight over a 23-week period until all rats had become obese. Further investigation of these differences found an altered lipometabolism, leptin-resistance, decreased expression of fasting-induced adipose factor (low levels induce deposition of triglycerides in adipocytes), and increased expression of fatty acid synthase in the gnotobiotic mice in comparison to the conventional mice. Investigation of this pathway and the discovery that obesity could be induced through an Enterobacter strain, but not a high fat diet, the clear role that microbes play in the development of obesity is made manifest [63]. Therefore, it is of the upmost importance to maintain gut balance, as the clear consequences of gut dysbiosis on an individual's weight and overall health have been demonstrated through numerous experiments.

\section{Antibiotics and the Microbiome Antibiotics elicit lasting effects on the gut flora.}

Broad-spectrum antibiotics targeting anaerobic bacteria elicit a disruption of the normal gut flora, and can do so for an extended period of time. One study demonstrated that recovery from broad-spectrum antibiotic use is only partial and can take up to four years to return to pre treatment states [64]. In an investigation in humans, it was found that just one course of antibiotics can produce changes in fecal bacteria content indicative of increased Clostridium difficile growth [65]. Further investigating the effects of antibiotics, one study discovered that a single course of antibiotics can disrupt over one-third of bacterial species in the gut. Monitoring this effect over time, it was discovered that many taxa returned to the pretreatment condition by 4 weeks from the termination of the treatment, however several taxa failed to recover after 6 months [66].

To further demonstrate the unwanted collateral damage antibiotics can have on the gut flora, one study showed that antibiotics not only killed bacteria, but also destroyed cells in the intestinal epithelium [67]. This is a serious side effect as intestinal epithelial cells play a crucial role in nutrient absorption and serves as part of our intestinal immune system, preventing translocation of bacteria from the gut into the bloodstream. Additionally, antibiotics were found to inhibit mitochondrial gene expression in mice, cascading to further damage of the epithelial cell lining of the gut [67].

\section{Broad-spectrum antibiotics disrupt gut microbial bal- ance.}

Broad- and narrow-spectrum antibiotics differ in their spectrum of activity, and therefore their effect on microbial floras throughout the body. While both broad- and narrow-spectrum antibiotics have the potential to alter the composition of the gut microbiome, it has been observed that broad-spectrum antibiotics are responsible for a larger effect in terms of normal flora alteration [29]. In regards to diversity, broad-spectrum antibiotics induce collateral damage by unintentionally reducing bacterial diversity in the gut, allowing for the expansion of low diversity pathogenic bacteria, an expansion and diversity profile that has been shown to be causative in the pathogenicity of obesity in numerous experiments $[61,62,68]$. Along these same lines, it has been found that broad-spectrum antibiotics, in particular, decrease the functional capacity and diversity of the gut microbiome, allowing for the expansion and collapse of certain indigenous taxa. This depletion of niche occupation in the gut allows for the proliferation of pathogenic bacteria that are often resistant to the effects of the antibiotics [68]. In a broad sense, broad-spectrum antibiotics reduce microbial diversity in the gut, thereby destroying the innate, mutualistic colonies of bacteria necessary for optimal function of our "secret organ".

A recent study found that the use of broad-spectrum antibiotics in conventional mice led to a spike in microbiota-liberated mucosal carbohydrates that literally serve as food for opportunistic pathogens to enhance their growth into the uninhabited microbial niches left from the course of antibiotics [69]. However, in an undisturbed gut, a wide diversity of gut microbes keeps op- 
portunistic pathogen proliferation contained through speciesspecies competition. Further investigations stemming from the previous study found that the introduction of Bacteroidetes, a symbiont in the gut, reduced free levels of mucosal carbohydrates in gnotobiotic mice, thereby limiting the growth of opportunistic pathogens such as Salmonella and C. difficile [69]. A similar study found that three different types of broad-spectrum antibiotics significantly reduced numbers of the targeted C. difficile, however they also unintentionally reduced the numbers of Firmicutes and Bacteroidetes, with a corresponding increase in the numbers of Proteobacteria [70]. Proteobacteria are a major phylum of bacteria that include a wide variety of pathogenic bacteria such as Escherichia, Salmonella, Vibrio, and Helicobacter [71]. Perhaps most significantly, recent research has shown that broad-spectrum antibiotics have a more significant effect on gram-positive species, such as Clostridium. These "Clostridial clusters" have been shown to favorably calibrate the immune system, and when antibiotics decimate their numbers, systemic inflammation results from an overactive immune response stemming from said immune disequilibrium. However, this same study found that immune equilibrium could be reestablished simply through the reintroduction of 46 Clostridial strains in the guts of mice [73].

Use of broad-spectrum antibiotics leads to significant reductions in bacterial diversity, taxonomic numbers, and equivalency of the proportions of bacterial phyla inhabiting the human gut [74-76]. One study noted that this decreased microbial diversity disrupts the crucial community relationships of not only bacteria, but also the other microbes that inhabit our gut [77]. The importance of maintaining bacteria diversity is paramount, as decreases in certain types of bacteria allows the proliferation of opportunistic pathogens due to the lack of microbial competition normally keeping these pathogenic bacteria at low numbers [78]. This presents an interesting paradigm shift, as normally non-pathogenic bacteria have the power to become pathogenic when microbial niches are cleared out from the use of broadspectrum antibiotics. A Clostridium difficile infection in is an example of opportunistic pathogen proliferation resulting from a lack of species-species competition that arises as a consequence of broad-spectrum antibiotic use [79]. Interestingly enough, the most effective treatment currently available to treat Clostridium difficile infections is a fecal transplant. This procedure involves transferring fecal matter from a healthy individual to a patient suffering from a Clostridium difficile infection in order increase the bacterial diversity of the gut through the restoration of beneficial bacteria killed off or suppressed from the course of broadspectrum antibiotics [80]. Current studies show that obese individuals have decreased intestinal microbial diversity in comparison to lean individuals $[81,82]$. Therefore, it is predicted that the increased use of broad-spectrum antibiotics in America is contributing to the current obesity trends, and states with higher antibiotic prescription rates should demonstrate higher obesity rates than those with lower levels of antibiotic prescriptions.

\section{CHAPTER II}

\section{Methods \\ Data Sources and Extraction \\ Behavioral Risk Factor Surveillance System (BRFSS)}

Established in 1984, the BRFSS is a cross-sectional telephone survey initiated by the Centers for Disease Control and Prevention (CDC) and is used by state health departments to collect prevalence data regarding health-related risk behaviors, chronic health conditions, and use of preventive services amongst U.S. residents 18 years of age or older. Respondent data from more than 500,000 adult interviews is tabulated by the CDC and published at the end of the year for each state. The BRFSS is currently the largest health survey system currently in place in the United States [83]. Data sets are publicly available at www. cdc.gov/brfss.

Data extracted from the BRFSS for analysis in this study included national and statewide obesity prevalence from 19932008 and from 2011-2014. Self-reported height and weight via the BRFSS are used to calculate Body Mass Index (BMI) of surveyed individuals, with a BMI greater than or equal to 30 distinguishing obesity. BRFSS measures of obesity tend to be relatively accurate, however studies have shown that obesity prevalence is most likely higher than indicated by the data due to the exaggeration of height and the under-reporting of weight of surveyed individuals [84].

\section{National Ambulatory Medical Care Survey (NAMCS)}

Established in 1973, the NAMCS is a national survey conducted by the National Center for Health Statistics (NCHS) for the CDC intended to collect objective, reliable information about the use of ambulatory medical care services in the United States. Data collected from this survey are based on a sample of visits to non-federal employed office-based physicians primarily engaged in direct patient care. Data is collected from the physician, rather than the patient, allowing for an expansion of information not possible in other NCHS surveys. Further methodology on the NAMCS is available from the NCHS [85].

Variables of interest from the NAMCS included the number of office visits by the physician's primary diagnosis and antimicrobial prescriptions ranging from 1993-2008. Specific categories of physician primary diagnosis included endocrine, metabolic, nutritional, and immune related disorders; infectious and parasitic diseases; and sleep disorders. Specific therapeutic classes of antimicrobials targeted for prescription totals included cephalosporins and penicillins. Level of specific drug use is reported in the NAMCS summary reports by total drug mentions. A drug mention is defined as documentation in a patient's record of a drug provided, prescribed, or continued at a visit (up to 10 per visit) [86]. Drug "mentions" and drug "use" were used interchangeably in this study, both representing the same value. It is also of importance to note that drug mentions and hospital visits, along with the corresponding standard error, are reported in thousands unless indicated otherwise. 


\section{Identification and Classification of Antibiotics}

Prescription drugs are classified and entered in the National Ambulatory Care Drug Database System by NCHS medication codes. Medication codes are linked to drug name, generic name, ingredient name, therapeutic effect, or by list of active ingredients. Prior to 2006, every drug reported in the National Ambulatory Medical Care Survey was assigned characteristics based on the Food and Drug Administration's National Drug Code Directory. However, in 2006, characteristics were changed in agreement with those outlined by the Lexicon Plus® database of Cerner Multum [87]. Drug categorizations reported in the NAMCS annual summaries from 1993-2008 were in agreement with the NCHS medication codes. Prescription data extracted from the NAMCS summary from 1993-2008 targeted the drug most prescribed within the therapeutic class of antibiotic. For penicillins this was Amoxicillin and for cephalosporins this was Ceftriaxone (Rocephin).

Antibiotic classifications based on spectrum of activity were done in accordance with expert opinion, as established in previous studies [88]. Broad-spectrum antibiotics were distinguished as amoxicillin-clavulanic acid, cephalosporins, macrolides, and fluoroquinolones. Narrow-spectrum antibiotics were distinguished as penicillin, amoxicillin, ampicillin, erythromycin, tetracycline, doxycycline, sulfonamides, and trimethoprim.

\section{QuintilesIMS Xponent Data}

The QuintilesIMS Xponent database is currently the most accurate measure of antibiotic prescriptions across the United States, capturing an estimated $100 \%$ of all outpatient prescription activity in the United States through a patented projection method [89]. Despite its accuracy, public use of this data is not readily available in the United States. However, recent efforts by the CDC have made this data available at a very limited level, ranging from 2011-2014 [90].

Variables of interest from the CDC-provided QuintilesIMS Xponent data and U.S. Census files included oral antibiotic prescriptions dispensed per 1000 population for the years 2011-2014. Reporting facilities comprising the presented prescription rates included all community and mail order pharmacies, but did not include federal healthcare facilities. Total national antibiotic prescriptions were comprised of 10 antibiotic classes including penicillins, macrolides, cephalosporins, fluoroquinolones, beta-lactams (increased activity), tetracyclines, trimethoprim-sulfamethoxazole, urinary anti-infectives, and lincosamides. Specific therapeutic classes of antibiotics targeted for state-to-state analysis included penicillin, macrolides, fluoroquinolones, and cephalosporins. Classifications of antibiotics are based on the QuintilesIMS Uniform System of Classification [91]. Along these parameters, beta-lactam antibiotics were distinguished as penicillins and cephalosporins.

\section{Statistical analysis}

Data from the NAMCS, BRFSS, and QuintilesIMS Xponent database were combined for analysis. Relationships between total antibiotic use on a national and state level were analyzed in comparison to national and state levels of obesity prevalence.
Analysis of antibiotic use included a breakdown into specific therapeutic categories to establish which classes were most significantly correlated with the state and national obesity rates. Statistical analysis of corresponding data used Microsoft Excel ${ }^{\circledR}$ to compute the Pearson product-moment correlation coefficient (r) and an associated p-value, with $\mathrm{p}<.0001$ considered statistically significant unless indicated otherwise. Correlation coefficients from the analysis were tabulated and graphed. To measure changes in antibiotic prescription rates and categories of primary diagnosis over time, a trend line was fitted to the data displaying the R-squared value as a measure of fit of the trend line. Error bars for class distinctions between antibiotics were calculated in accordance with NCHS recommendations. Timeframes targeted for analysis in this study were determined by the availability of antibiotic prescription data for those years.

\section{Chapter III}

\section{Results \\ State-distinguished antibiotic use and obesity rates show similar patterns of distribution.}

Antibiotic prescriptions dispensed in U.S. community pharmacies per 1000 population decreased from 877 in 2011 to 835 in 2014. However, an individual comparison of states shows noteworthy differences in the amount of prescriptions dispensed over this time period by each state. Similar patterns in self-reported adult obesity (18 years $\geq$ ) were observed over this same period (Figure 1).

Over the study period (2011-2014), state obesity rates were positively correlated with the total amount of antibiotics prescribed in each state. Further analysis indicated variance between individual therapeutic antibiotic classes and relationships with the state obesity rate. Cephalosporin prescription rates demonstrated the strongest relationship with the variance in state obesity rates for all four years of the study period (20112014). All correlations were found to be significant $(\mathrm{p}<.0001$; Figure 2).

All correlations were significant $(\mathrm{p}<.0001)$. Error bars indicate $95 \%$ confidence intervals. Beta-lactam category includes penicillins and cephalosporins. All classes category includes penicillins, macrolides, cephalosporins, fluoroquinolones, betalactams (increased activity), tetracyclines, trimethoprim-sulfamethoxazole, urinary anti-infectives, and lincosamides. Therapeutic classes are based on the QuintilesIMS Uniform System of Classification. See Table 2 (Appendix) for tabulated values.

*=indicates strongest correlation for a given year.

\section{Beta-lactam Prescriptions and Obesity.}

Distinguished as the therapeutic class most strongly correlated with state obesity rates, cephalosporin prescription rates were tracked in an expanded time period (1993-2008) to investigate correlated changes with the obesity rate. Additionally, penicillin prescription rates were tracked over this period to provide comparison to another type of antibiotic in the beta-lactam therapeutic class, with the intention of highlighting characteristic 


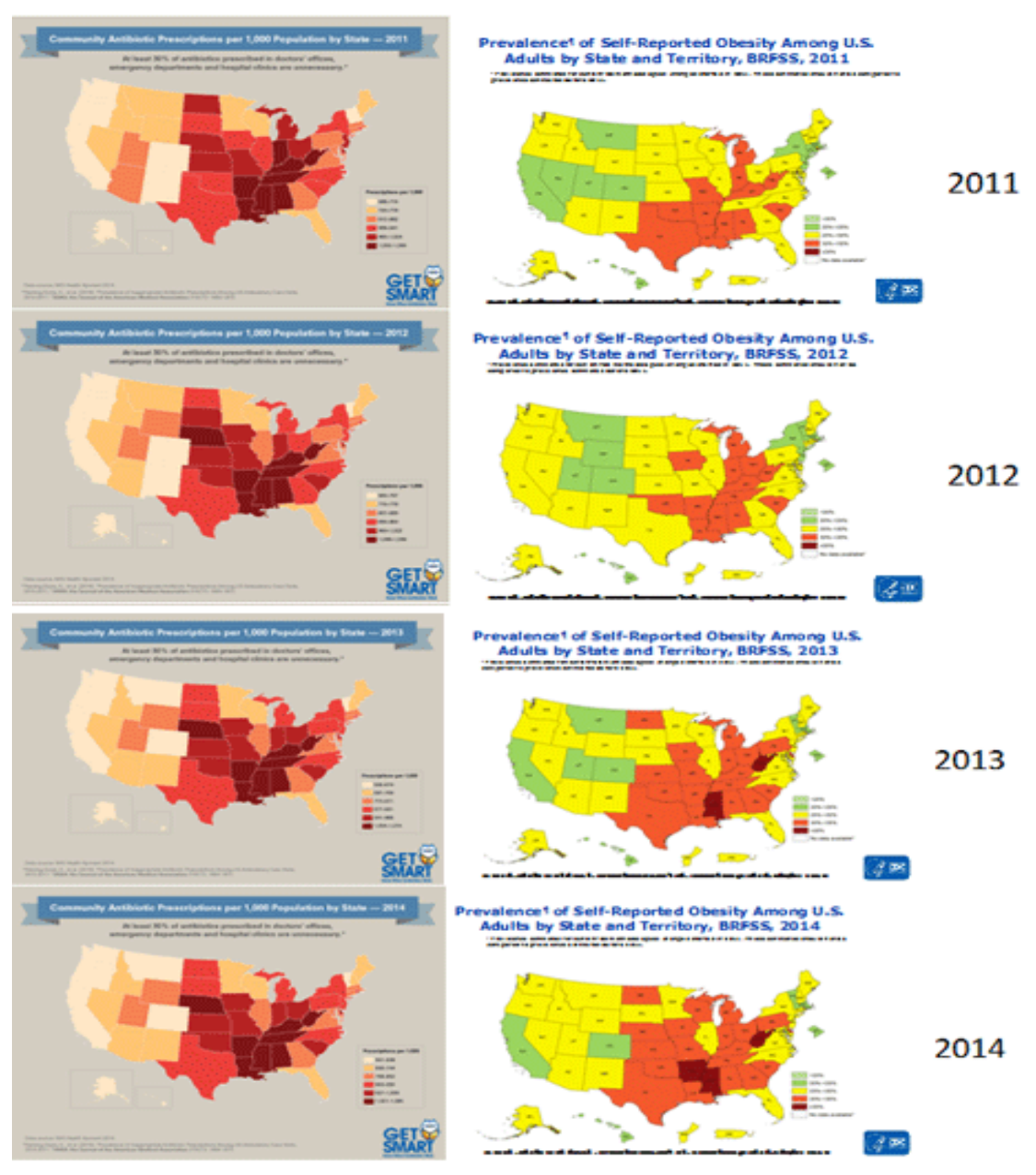

Figure 1:Comparison of prevalence maps for community antibiotic prescriptions per 1,000 population by state and self-reported obesity by state. Outpatients aged 18 and older, U.S. 2011-2014.

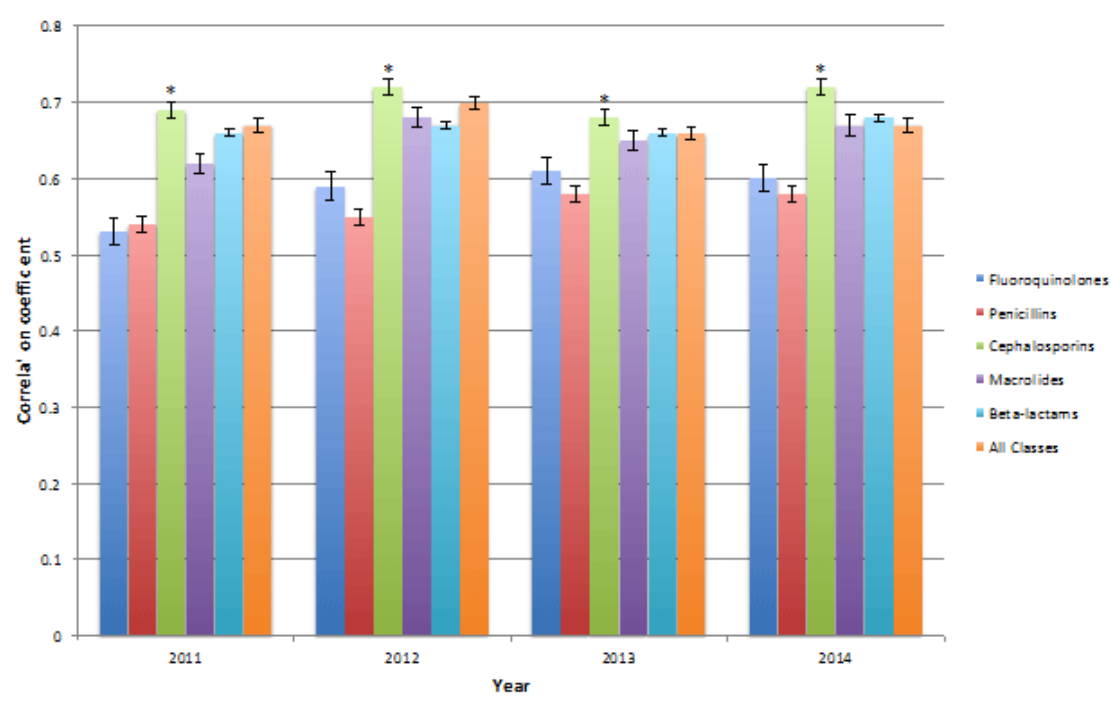

Figure 2: Graphical representation of correlations between state antibiotic prescriptions per 1,000 population and self-reported obesity rate by state. 
Table 2: Correlation coefficient ( $r$ ) and associated p-value for stateassociated antibiotic prescriptions per 1000 people and self-reported obesity rate (2011-2014).

\begin{tabular}{|c|c|c|}
\hline & Correlation Coefficient (r) & P Value \\
\hline \multicolumn{3}{|l|}{2011} \\
\hline Fluoroquinolones & .53 & 7.17E- $05^{* *}$ \\
\hline Penicillins & .54 & $4.29 \mathrm{E}-05^{* *}$ \\
\hline Cephalosporins & $.69^{*}$ & $1.96 \mathrm{E}-08^{* *}$ \\
\hline Macrolides & .62 & $9.09 \mathrm{E}-07^{* *}$ \\
\hline Beta-lactams ${ }^{i}$ & .66 & $1.48 \mathrm{E}-07^{* *}$ \\
\hline All Classes $^{+}$ & .67 & $6.91 \mathrm{E}-08^{* *}$ \\
\hline
\end{tabular}

2012

\begin{tabular}{|c|c|c|}
\hline Fluoroquinolones & .59 & $5.66 \mathrm{E}-06^{* *}$ \\
\hline Penicillins & .55 & $3.29 \mathrm{E}-05^{* *}$ \\
\hline Cephalosporins & $.72^{*}$ & $2.42 \mathrm{E}-09^{* *}$ \\
\hline Macrolides & .68 & $4.95 \mathrm{E}-08^{* *}$ \\
\hline Beta-lactams $^{\mathrm{i}}$ & .67 & $6.67 \mathrm{E}-08^{* *}$ \\
\hline All Classes $^{+}$ & .70 & $1.71 \mathrm{E}-08^{* *}$ \\
\hline
\end{tabular}

\section{3}

\begin{tabular}{|c|c|c|}
\hline Fluoroquinolones & .61 & $2.56 \mathrm{E}-06^{* *}$ \\
\hline Penicillins & .58 & $1.11 \mathrm{E}-05^{* *}$ \\
\hline Cephalosporins & $.68^{*}$ & $5.24 \mathrm{E}-08^{* *}$ \\
\hline Macrolides & .65 & $2.81 \mathrm{E}-07^{* *}$ \\
\hline Beta-lactams & .66 & $1.77 \mathrm{E}-07^{* *}$ \\
\hline All Classes $^{+}$ & .66 & $1.25 \mathrm{E}-07^{* *}$ \\
\hline
\end{tabular}

2014

\begin{tabular}{|c|c|c|}
\hline Fluoroquinolones & .60 & $4.00 \mathrm{E}-06^{* *}$ \\
\hline Penicillins & .58 & $1.12 \mathrm{E}-05^{* *}$ \\
\hline Cephalosporins & $.72^{*}$ & $2.45 \mathrm{E}-09^{* *}$ \\
\hline Macrolides & .67 & $8.16 \mathrm{E}-08^{* *}$ \\
\hline Beta-lactams $^{\mathrm{i}}$ & .68 & $5.18 \mathrm{E}-08^{* *}$ \\
\hline All Classes $^{+}$ & .67 & $7.86 \mathrm{E}-08^{* *}$ \\
\hline
\end{tabular}

differences between the two drugs. Based on the availability of data from the NAMCS summaries, the most frequently mentioned generic equivalent of the specific therapeutic class of beta-lactam antibiotic was used as a representative sample for the class as a whole. The most frequently mentioned generic equivalents were
Ceftriaxone (Rocephin) for cephalosporins and Amoxicillin for penicillins. Trend analysis over this period found a strong relationship between Ceftriaxone drug mentions and national prevalence of obesity ( $r=.96, p=6.07 \mathrm{E}-9$; Figure $3 \mathrm{~A}$ ), while lacking to demonstrate the same strength of relationship for Amoxicillin drug mentions ( $\mathrm{r}=.18, \mathrm{p}=.525$; Figure $3 \mathrm{~B}$ ) Over this period, Ceftriaxone increased by $110.7 \%$ from 1559 mentions ( $\mathrm{s}=147$ ) in 1993 to 3284 mentions ( $s=210$ ) in 2008, while Amoxicillin remained relatively stable, showing a $.91 \%$ increase from 3532 mentions ( $s=312$ ) in 1993 to 3564 mentions ( $s=276$ ) in 2008 (Figure 3).

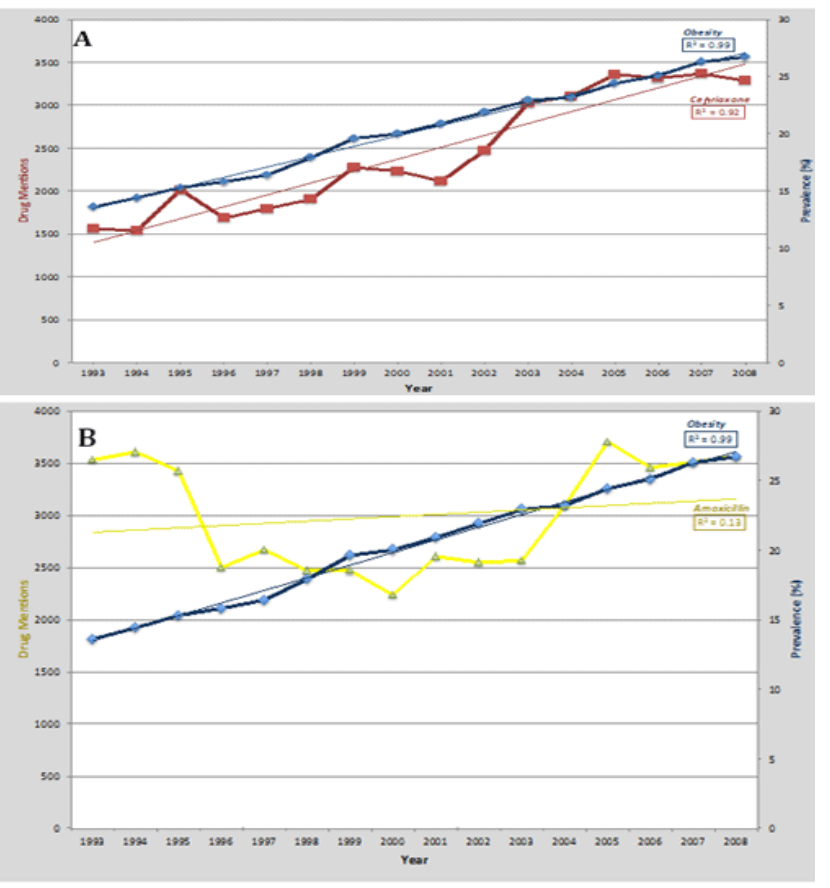

Figure 3: Trends in (A) Ceftriaxone and (B) Amoxicillin drug mentions and self-reported obesity in the United States (1993-2008).

Drug mention data is from NAMCS Emergency Department summaries and self-reported obesity data are from the BRFSS. Drugs names are based on Multum Lexicon terminology (drug name reflects the active ingredients of a drug).

\section{Cephalosporin use poses potential adverse side effects.}

Proposed risks with antibiotic use were tracked from 1993 to 2008 and were based on NAMCS summary data. Over this period, number of hospital visits for sleep disorders showed a $483.7 \%$ increase from 1.47 million in 1993 to 8.58 million in 2008. Although Ceftriaxone use demonstrated a significant, positive relationship with hospital visits for sleep disorders ( $r=.94$, $\mathrm{p}<.0001$; Figure 4A), Amoxicillin use did not ( $\mathrm{r}=.24$, n.s.; Figure 4A). Hospital visits for endocrine, nutritional, metabolic diseases and immunity disorders (NEMIDs) showed a $121.9 \%$ increase over the study period, increasing from 25,428 visits in 1993 to 56,417 visits in 2008. Ceftriaxone use over this period showed a significant, positive relationship with associated NEMID hospital visits $(r=.83, p<.0001$; Figure $4 B)$ whereas Amoxicillin use 

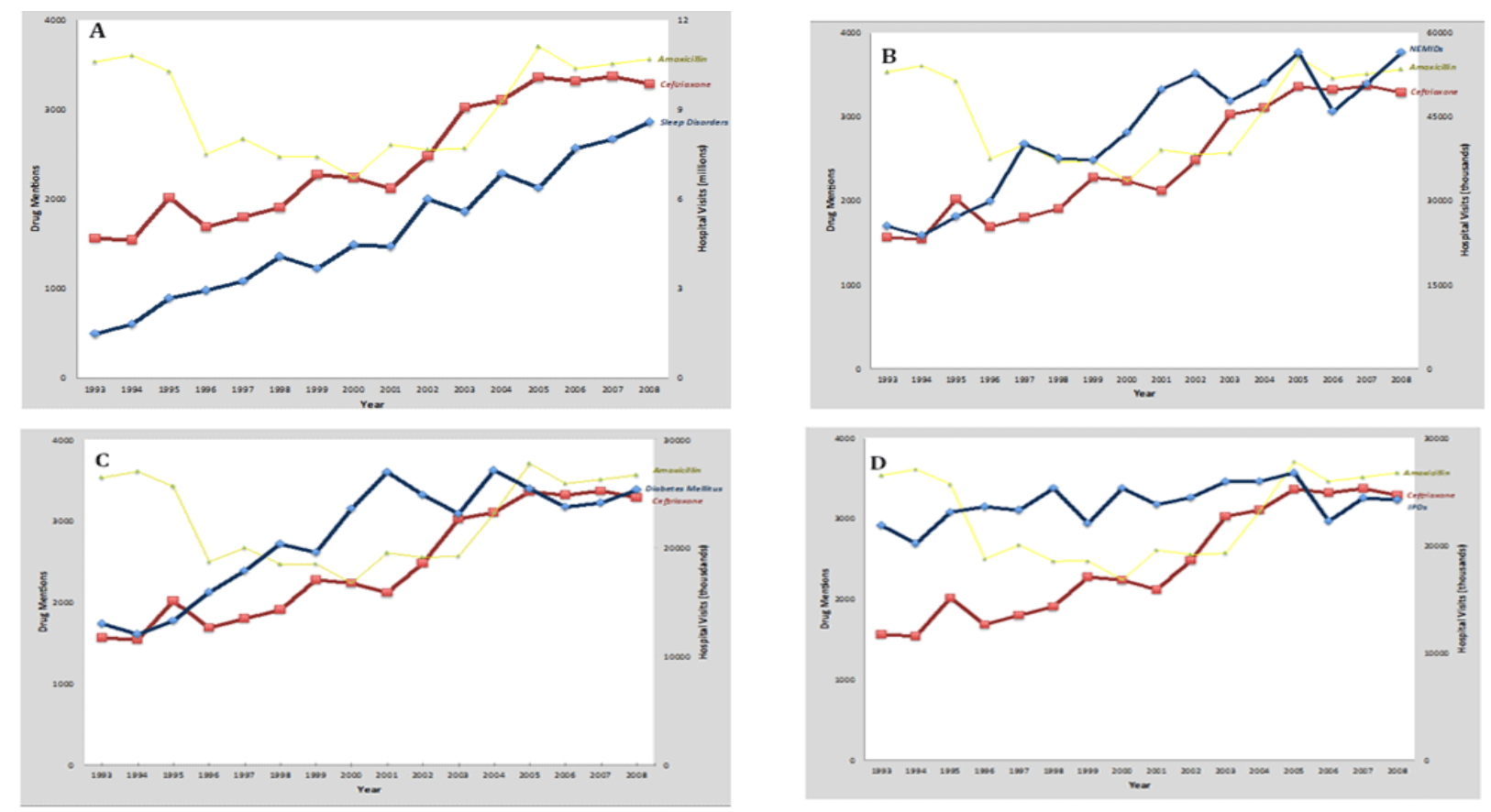

Figure 4: Trends in Ceftriaxone and Amoxicillin drug mentions and physician primary diagnosis of (A) sleep disorders; (B) endocrine, nutritional, metabolic diseases and immunity disorders (NEMIDs); (C) diabetes mellitus; and (D) infectious and parasitic diseases (IPDs).

did not ( $r=.002$, n.s.; Figure 4B). Hospital visits for diabetes over this period increased by $95.2 \%$ from 12,997 in 1993 to 15,365 in 2008. Ceftriaxone drug mentions over this time period showed a positive, significant relationship with hospital visits for diabetes mellitus ( $\mathrm{r}=.77, \mathrm{p}<.001$; Figure 4C), however Amoxicillin use did not ( $r=-.15$, n.s.; Figure 4C). Hospital visits for infectious and parasitic diseases (IPDs) showed a $10.9 \%$ increase from 21,828 visits in 1993 to 24,213 visits in 2008. A moderately positive relationship was found with Ceftriaxone use ( $\mathrm{r}=.54, \mathrm{p}<.05$; Figure 4D), but not with Amoxicillin use ( $r=-.24$, n.s.; Figure 4D).

Time period of study is 1993-2008. Drug mention and physician primary diagnosis data are from NAMCS Advanced Data summaries.

\section{Chapter IV}

\section{Discussion/Conclusion}

Antibiotic use is related to state differences in obesity.

A crude comparison of state-distinguished antibiotic use and obesity rates show similar patterns of distribution from 2011 to 2014 (Figure 1). When this distribution was further broken down into individual therapeutic classes of antibiotics, it was discovered that cephalosporin prescriptions per 1000 population in each state demonstrated the strongest correlation with the associated state obesity rate (Figure 2; Table 2) Cephalosporins are part of a broader class of antibiotics called beta-lactams. This therapeutic class functions through competitive inhibition of the transpeptidase enzyme, thus preventing it from cross-linking peptidoglycan units, resulting in disrupted bacterial cell wall synthesis [91]. Other antibiotics in this broader class include penicil- lins, monobactams, and carbapenems.

For this study, penicillins were considered separately from cephalosporins and in conjunction with cephalosporins as part of the beta-lactam class in regards to comparison of antibiotic prescriptions and state obesity prevalence. Over the study period (2011-2014), it was discovered that penicillins demonstrated the lowest average correlation with state obesity prevalence out of all antibiotics considered, despite having the same structural, pharmacological, and mechanistic action as cephalosporins. By comparison, cephalosporins consistently showed the strongest relationship with state obesity rates over the study period [92]. Further investigation of this difference revealed a potential underpinning for the observed correlational differences. Penicillins are a class of antibiotics considered narrow in their spectrum of activity, primarily targeting gram-positive bacteria. However, cephalosporins are considered broad in their spectrum of activity and can elicit potentially harmful collateral damage on the normal gut flora due to their effect on both gram-positive and gram-negative bacteria throughout the body [93]. While corelational results do not imply causation, it is interesting to note how the change from a broad- to narrow-spectrum drug in the same mechanistic class of antibiotics can significantly reduce the relationship with obesity prevalence. This suggests that the increased spectrum of activity can have severe consequential side effects through disruption of the normal gastrointestinal flora, manifesting is a rather morbid fashion through increased state obesity rates. The increased collateral damage on GI flora brought on by a course of broad-spectrum antibiotics has been noted in multiple studies, while failing to find the same effect from narrow-spectrum antibiotic use [94-96]. 
Obesity rates demonstrate a stronger relationship with cephalosporin drug mentions than amoxicillin (1993-2008).

The notable difference between cephalosporin and penicillin prescriptions with state obesity rates prompted an expanded investigation of the use of these beta-lactam antibiotics and associated obesity rates on a national scale. For this expanded study period (1993-2008), the prescription levels of the most frequently mentioned cephalosporin and penicillin were tracked. Drug mentions are defined by the NAMCS as documentation in a patient's record of a drug provided, prescribed, or continued at a visit [97]. For this time period, Ceftriaxone was the most frequently mentioned cephalosporin and Amoxicillin was the most frequently mentioned penicillin.

\section{Pharmaceutical Characteristics of Ceftriaxone and Amoxicillin.}

Cephalosporins are classified by "generation" which denotes spectrum of antimicrobial activity. As cephalosporins increase in generations, their spectrum of activity increases, with an increased effect on gram-negative bacteria and increased beta-lactamase stability. Ceftriaxone is a third-generation cephalosporin (3gc) considered broad in its spectrum of activity due to its effect on both gram-positive and gram-negative bacteria [98].

Amoxicillin is considered an aminopenicillin within the penicillin class [99]. Amoxicillin is considered narrow in its spectrum due to its high susceptibility of degradation by betalactamase-producing bacteria $[99,100]$. Due to this susceptibility, Amoxicillin is generally prescribed with Clavulanic acid, an inhibitor of beta-lactamase enzymes, becoming broad-spectrum in this combination [100]. However, for this study, Amoxicillin was administered without Clavulanic acid, thereby rendering its spectrum narrow due to the high level of microorganisms resistant to its mechanistic effects [101].

\section{Ceftriaxone demonstrates a strong relationship with the national obesity rate over time.}

Comparison of Ceftriaxone and Amoxicillin serves useful in the understanding of how broad and narrow-spectrum antibiotics differ in their associated correlations with the obesity rate due to their similar classification as beta-lactam antibiotics. Over this period, it was discovered that Ceftriaxone correlated very strongly with the obesity rate $(r=.96, p<.0001$; Figure $3 A)$, whereas Amoxicillin showed a weak correlation $(r=.18, p=.53$; Figure 3B). Numerous studies have found that an altered gastrointestinal flora is associated with obesity [102-104]. Therefore, despite the lack of causality power provided by correlations, the use of obesity as a marker of microbial disturbance allows for a rudimentary understanding of the potential severity of broadspectrum antibiotic induced side effects on normal microbial flora when compared to narrow-spectrum antibiotics.

\section{Ceftriaxone drug mentions are strongly correlated with hypothesized side effects of broad-spectrum antibiotic use.}

A mechanism explaining the association between Cef- triaxone use and the proposed side effects will be discussed in section 4.4.

Ceftriaxone drug mentions demonstrate a positive relationship with hospital visits for sleep disorders.

From 1993-2008, Ceftriaxone drug mentions demonstrated a strong relationship with hospital visits for sleeping disorders ( $\mathrm{r}=.94, \mathrm{p}<.0001$; Figure $4 \mathrm{~A}$ ), whereas, Amoxicillin did not $(\mathrm{r}=.24$, n.s.; Figure 4A). Maintaining interpretations within the realm of broad- and narrow-spectrum antibiotic comparison, it is clear that an interaction is occurring between increased spectrum of antibiotics and sleep disorders. The proposed mechanism will explore the co-relational differences in light of inflammation and neuroendocrine irregularities.

Ceftriaxone drug mentions demonstrate a positive relationship with hospital visits for endocrine, nutritional, metabolic diseases, immunity disorders (NEMIDS) and diabetes mellitus.

From 1993-2008, hospital visits for NEMIDs showed a strong, positive relationship with Ceftriaxone mentions $(\mathrm{r}=.83$, $\mathrm{p}<.0001$, Figure 4B) whereas Amoxicillin mentions did not ( $r=.002$, n.s.; Figure 4B). Examples of NEMIDs include disease of endocrine and thyroid glands; nutritional deficiencies; disorder of lipid, amino acid, mineral, and carbohydrate metabolism; acidbase imbalances; and disorders involving immune mechanism. Further coding of NEMIDs can be found in CDC codings contained in ICD-9 [105]. Over this same time period, it was found that hospital visits for diabetes mellitus was strongly related with Ceftriaxone mentions ( $r=.77, \mathrm{p}<.001$; Figure $4 \mathrm{C}$ ), however no significant relationship was found with Amoxicillin ( $r=-.15$, n.s.; Figure 4C). As noted before, the suspected collateral damage stemming from a course of broad-spectrum Ceftriaxone in comparison to narrower spectrum Amoxicillin is highlighted, with additional evidence supporting the hypothetical side effects (discussed in 4.4).

Ceftriaxone drug mentions were not associated with a decrease in hospital visits for infectious and parasitic diseases.

From 1993-2008, hospital visits for infectious and parasitic diseases (IPDs) increased by $10.9 \%$ and demonstrated a positive relationship with Ceftriaxone mentions $(r=.54, p<.05$; Figure 4D). However, a negative relationship with Amoxicillin mentions was discovered ( $\mathrm{r}=-.24$, n.s.; Figure 4D). These results prove interesting as antibiotic prescriptions are expected to show negative associations with hospital visits for infectious and parasitic diseases. However, it is key to note that while Ceftriaxone mentions were positively correlated with IPDs, Amoxicillin showed a weak, but negative association. This result provides an interesting area of investigation, as Amoxicillin, a narrow-spectrum antibiotic, appears to have increased effectiveness in treating IPDs than does Ceftriaxone, a broad-spectrum antibiotic. Additionally, Amoxicillin use was not significantly correlated with any of the proposed adverse effects of antibiotic use, suggesting a limited collateral effect on our GI flora (Figure 4A-D). Despite the limited causality 
power provided by correlations, if these associations hold true in an experimental format, perhaps broad-spectrum antibiotics are not as effective as current knowledge posits, and the current trend in increased broad-spectrum antibiotic use is doing us substantially more harm than good. If narrow-spectrum antibiotics prove to be more effective at treating IPDs, then that points a finger to broad-spectrum antibiotics as a possible contributing culprit for many of the chronic disease patterns currently occurring in the United States.

However, it is also important to consider resistance as an explanation for these patterns. Current studies and clinical observations have noted the increased resistance of gram-negative bacteria in response to third generation cephalosporin use, such as Ceftriaxone. When a course of cephalosporins is administered, a unique niche opening is given to these opportunistic, gramnegative bacteria. Due to the spectral nature of third-generation cephalosporins, the commensal gram-positive bacteria inhabiting the mucosal layer of the GI track remain susceptible, giving the increasing number of resistant gram-negative bacteria the opportunity to rapidly expand into niches vacated by wiped out grampositive bacteria communities. This opportunistic growth allows normally non-threatening gram-negative bacteria to develop a pathogenic presence. Amoxicillin also suffers from a high level of microbial resistance, however, gram-positive bacteria tend to be resistant, thereby keeping opportunistic pathogens in check. In fact, due to this resistance, Amoxicillin is rarely prescribed without Clavulanic acid in current practices. The narrowed spectrum of Amoxicillin leaves gram-negative bacteria relatively undisturbed, preventing the opportunity for these species to develop resistance. Additionally, the increased resistance of gram-positive bacteria, most of which are commensal and make up the mucosal lining of the gut, prevents the niche vacation associated with broad-spectrum antibiotic use, thereby preventing opportunistic gram-negative growth.

\section{Proposed mechanisms of antibiotic-induced obe- sity Broad-spectrum antibiotic use elicits gut dysbiosis.}

Insight regarding a pathway for the manifestation of obesity and hypothetical side effects associated with broadspectrum antibiotic use finds its cornerstone at gut dysbiosis. Dysbiosis refers to an alteration of the endogenous flora in our gut, predisposing an individual to microbial overgrowth and a loss of normal gut function. This microbial imbalance in the gut is mechanistically linked to a disruption of metabolic and immune response pathways throughout the body [106, 107]. To best understand the significance of this effect, it is useful to view the gastrointestinal tract as a rainforest, containing an abundance of microbial species, each inhabiting a unique niche, with community ecology playing a crucial role for overall function. Maintenance of a stable, balanced, and diverse microbial community within the gut is absolutely critical for overall health. When this proper microbial balance is disturbed, a significant amount of "niche opening" occurs, allowing for the pathogenic overgrowth of opportunistic species both commensal and inherently pathogenic in nature. One study demonstrated this effect, showing that a dis- rupted gut flora contained significantly less species diversification when compared to a "healthy" gut due to opportunistic overgrowth of microbial species [108]. Aristotle noted, "Virtue...is a mean between two kinds of vice, one of excess and the other of deficiency" [109]. Paralleling this ideology, a balanced gut should avoid any behavior promoting unbalanced growth in either realm of excess or deficiency. Unfortunately, severe disturbance of the endogenous gut flora can be brought about through a variety of different factors including, but not limited to, dietary composition, stress, and antibiotic therapies [110]. In accordance with the results of this study, antibiotic therapies will be targeted in the mechanistic focus.

Returning to the view of the gut as a diverse ecosystem, antibiotics can be equated to fire. However, not all antibiotics burn a flame of the size. Broad-spectrum antibiotics enter the gut with the power of a flamethrower, capable of evoking an enormous disruption of species balance, allowing for overgrowth of opportunistic species into vacated niches normally inhabited by commensal microbes. In contrast, narrow-spectrum antibiotics can barely even find our rainforest, and if they do, they enter with the power of a single match, causing little to no effect on our microbial composition. Within the realm of broad-spectrum flamethrowers, this study warrants a particular attention to Ceftriaxone, and by appropriate comparison, Amoxicillin, match-like in its effect, and contained within the realm of narrow-spectrum antibiotics.

Ceftriaxone is properly classified as a third-generation cephalosporin (3gc), a class of antibiotics capable of procuring enormous disruptions to the endogenous gastrointestinal (GI) communities due to their spectrum of activity. Additionally, drugs in this antimicrobial class, have been notoriously noted for their selective encouragement of the rapid overgrowth of multi-drug resistant bacteria, unable to be eliminated or inhibited by further antimicrobial therapy [111-113]. If 3gc use continues at current levels, the proliferation of resistant, pathogenic gram-negative organisms could destroy current methods of infectious disease treatment, bringing dire consequences for human health with it.

Third-generation cephalosporin-associated microbial overgrowth is not solely limited to inherently pathogenic species, as many commensal species can become pathogenic when allowed uninhibited sites for colonization [114-116]. As the saying goes, "the dose makes the poison." [117]. For example, coagulasenegative staphylococci, Pseudomonas aeruginosa, and Candida albicans are all normally commensal organisms that can develop pathogenic qualities in the instance of 3gc use [118-120]. These microbial imbalances are often of more concern than inherently pathogenic organisms, as infectious signs of inherently pathogenic microbes are commonly known and readily identified, whereas infectious signs of commensal microbe overgrowth can go undetected or be symptomatically assigned to a different etiology. By comparison, some inherently pathogenic organisms prone to 3gc-induced overgrowth due to their remarkable resistance to this class of drugs are Clostridium difficile, penicillin-resistant pneumococci, and methicillin-resistant Staphylococcus aureus (MRSA) [111]. 
Evidence of antibiotic-induced states of overgrowth and dysbiosis often quickly manifest through opportunistic bacterial or yeast infections. For example, Enterococcal bacteremia is often noted to develop following cephalosporin use, indicative of cephalosporin-resistant Enterococcal colonies expanding beyond their normal limits and into the blood [121]. Additionally, in controlled human trials, individuals given a cephalosporin developed Candidiasis, a systemic infection of a normally commensal fungus, within 2 to 3 days following treatment, indicating Candida overgrowth into vacated niches [122]. However, in a separate study, individuals given amoxicillin showed no increase in yeast colonization while similar experimental conditions were maintained [123]. These results support the study's hypothesis, claiming that broad-spectrum antibiotics elicit a greater disturbance of non-targeted GI flora than do narrow-spectrum antibiotics, thereby presenting an increased risk for metabolic- and immunerelated side effects. Resistant organisms are of particular interest to an understanding of 3gc-induced microbial shifts, as resistance allows overgrowth into GI niches vacated by susceptible species. Therefore, this model of resistance-permitted overgrowth is key to understanding the development of gut dysbiosis and its direct effects on gut function.

\section{Broad-spectrum induced gut dysbiosis causes in- creased intestinal permeability and low-grade, sys- temic inflammation.}

Sitting at one of the most dramatic and cascading mechanistic cornerstones involved in gut health is intestinal permeability. The intestinal wall is composed of four layers and serves a crucial role as the first mechanism of defense for the GI immune system. The individual layers of the intestinal wall are the mucosa, submucosa, muscularis propria, and the serosa. The mucosa layers serves as the most important for absorptive function, made up of a single layer of epithelial cells, connected by tight junctions. Epithelial cells are crucially responsible for keeping intestinal bacteria and their associated toxins contained inside the gut and out of contact with the rest of the body. Underneath the mucosa layer, the submucosa consists of a variety of inflammatory and lymphatic cells, autonomic nerve fibers, and ganglion cells, which play a role in immune function and gut-brain communication [124]. The intestinal epithelium lining is kept intact primarily by a group of three gram-positive, commensal bacteria belonging to the genus of Clostridium, Lactobacillus, and Enterococcus [42]. These intestinal symbionts communicate through the secretion of chemical metabolites, allowing for the maintenance of intestinal wall integrity. One example of the interplay between these species and intestinal epithelium involves the production of a metabolite called 3-propionic acid (IPA) by Clostridium sporogenes [125]. These metabolites function by binding to the pregnane $\mathrm{X}$ receptor (PXR) inside the nucleus of intestinal epithelial cells. This binding activates the pregnan $\mathrm{X}$ receptor (PXR) inside the nucleus of intestinal epithelial cells, leading to the activation of junction protein-coding mRNAs. Tight junction proteins are responsible for maintaining epithelial integrity via strengthening of the connection between adjacent epithelial cells of the mucosal lining. Additionally, PXR activation results in the suppression of tumor necrosis factor alpha (TNF- $\alpha$ ), a pro-inflammatory cytokine contained within the submucosa [126].

Unfortunately, these intestinal symbionts are very susceptible to antibiotic treatment, especially that of third-generation cephalosporins [113]. 3gc administration results in the partial depletion of commensal, mucosal bacteria, which are responsible for preventing intestinal bacteria from passing through the intestinal wall and into the bloodstream. The deleterious effect of intestinal symbiont death was demonstrated in a study showing that a disruption of IPA and PXR signaling led to a "distinctly leaky intestinal epithelium pathology" [126]. Additionally, an absence of IPA led to the increased proliferation of enterocytemediated inflammatory cytokine TNF- $\alpha$ [126]. Further studies have found that the endogenous lectin complement pathway also plays a key role in the initiation of intestinal inflammation, which is normally mediated by an intact epithelium [127]. Not only do $3 \mathrm{gc}$ kill off commensal microbes, whose metabolic byproducts improve tight junctions between epithelial cells, but this damage also induces further intestinal inflammation, thereby demonstrating the unfavorably synergistic way $3 \mathrm{gc}$ use can affect the gut.

The depletion and subsequent loss of mucosal-maintaining metabolites can lead to increased intestinal permeability, characteristically described as Leaky Gut Syndrome. This syndrome indicates that damage to the intestinal lining has caused it to become increasingly porous, allowing for toxins, waste, and undigested food molecules to freely flow into the bloodstream [128]. Perhaps the most dangerous content to leak from the gut into the bloodstream is bacterial Lipopolysaccharide (LPS). LPS is a heat stable endotoxin, serving as the major component of the outer membrane of gram-negative bacteria. LPS is composed of a polysaccharide chain, an oligosaccharide chain, and an immunostimulatory lipid A section [129]. When Lipid A of LPS is encountered by the host immune system, a strong immune response is initiated, leading to low-grade systemic inflammation [130]. LPS is a potent pro-inflammatory cytokine inducer, being deemed the triggering inflammatory factor causative of the onset of insulin resistance, obesity, and diabetes [131]. The immune response mounted to LPS is initiated by pro-inflammatory cytokines, with increased TNF- $\alpha$ and interleukin (IL)-1ß mRNA expression in less than an hour from the initial exposure [132]. This immune response invariably results in low-grade, systemic inflammation, which has been termed, "the silent fire", due to its relatively unnoticeable effects on a macroscopic level until it reaches a chronic state [133]. Current studies have found that increased plasma levels of these pro-inflammatory cytokines are increased in the insulin resistant states of obesity and type 2 diabetes [134]. The proliferation of TNF- $\alpha$ in particular has been shown to promote additional pro-inflammatory cytokine release and an associated reduction in anti-inflammatory cytokines, such as adiponectin [135]. While low grade, systemic inflammation is a necessary part of a healthy immune response, when allowed to persist at a chronic level, this "silent fire" can cause enormous detriments to health, predisposing an individual to many chronic diseases. 


\section{Low-grade systemic inflammation is a precursor for chronic disease.}

The death of significant portions of our intestinal symbionts leads to a damaged intestinal epithelium, overgrowth of opportunistic pathogens, and subsequent submucosal inflammation. These factors collectively contribute to intestinal hyper-permeability, which allows intestinal contents passage into the blood stream. Detection of intestinal contents in systemic circulation results in a cytokine-mediated immune response that promotes the development of low-grade, systemic inflammation.

Pro-inflammatory cytokines have a variety of harmful effects on hormones and neurotransmitters directly tied to metabolic function, sleep, and eating behaviors. Their relatively quick activation and sustained proliferation following LPS exposure is concerning, however, the cycles of resistance and physiological malfunction resulting from their cyclic activation proves to be of more pressing interest [132]. Crucial to understanding the manifestation of their effects, the molecular targets of pro-inflammatory cytokines must be highlighted.

Pro-inflammatory cytokines alter the metabolism of monoamines, including norepinephrine, serotonin, and dopamine [136]. Abnormal levels of these neurotransmitters have been linked to mood, sleep, and metabolic disorders [136-138]. The strong correlation of Ceftriaxone with sleep disorders, obesity, and diabetes indicated by the results of this study seem to be better understood in light of this cytokine-mediated mechanism resulting from broad-spectrum induced gut dysbiosis (Figure 3A; 4A,C). Pro-inflammatory cytokines have also been noted to activate Corticotrophin-Releasing Hormone (CRH), which has a strong stimulatory effect on the Hypothalamic-Pituitary-Adrenal axis (HPA axis), a complex feedback loop between three major endocrine glands responsible for hormone production. As a potential manifestation of these irregular hormonal activation, studies have found increased levels of CRH in patients with depressive behavior, altered appetite, and disrupted sleep patterns [139]. Studies have also found that alterations to the bodyweight/ appetite/satiety set point determined by the hypothalamus is altered by increased levels of cytokines and activation of the HPA axis by CRH [140]. Along with the stimulatory effects they have on the HPA axis, pro-inflammatory cytokines illicit glucocorticoid resistance through direct interaction with glucocorticoid receptors [136]. This interaction leads to an induction of glucocorticoid hormonal resistance in nervous, endocrine, and immune system tissues prompting disorder and disease of these systems [141]. This helps provide mechanistic understanding to the notable correlations found in this study (Figure 4B). Additional studies have found a persistence of increased levels of plasma pro-inflammatory cytokines in the insulin resistant states of obesity and type II diabetes. This suggests that low-grade, systemic inflammation in response to LPS, persists over a chronic period of time, silently burning, until it manifests as a chronic disease [142]. From a mechanistic point of view, once an individual reaches this state, a vicious cycle of metabolic and immune imbalance is entered, which can promote the development of a variety of chronic conditions.
The pathophysiology of obesity is undoubtedly complex, involving the interaction of numerous pathways and signaling molecules, each with a variety of factors contributing to their circulating level. Tracing the interplay of cytokines, hormones, and neurotransmitters in regards to the development of obesity provides a mechanistic complexity, as a seemingly infinite number of interactions and effects directly related to human disease can take place. In no way is a conclusion regarding an understanding of the progression of obesity in totality being offered at this point. However, by starting a mechanistic search for obesity at the end point, and tracing it back along its known causal pathways in association with related conditions, the GI biome frequently serves as a mechanistic stepping-stone that can frequently be called home. At a minimum, evidence of the threads connecting humans directly to their microbial world should prompt behavior avoidant of dysbiosis and pursuing microbial harmony. However, one way this harmony is guaranteed disruption is through the use of broad-spectrum antibiotics. The resulting dysbiosis has clearly been shown to induce serious consequences for health, many of which have been demonstrated through an evidence-backed mechanism and the relationships found in this study (Figure 4AD).

\section{Pharmaceutical approaches to maintain gut health.}

\section{Antibiotics demonstrating high gram-negative re- sistance and low gram-positive resistance should be avoided.}

As the results of this study suggest, not all antibiotics are created equal. Many antibiotics are incredibly beneficial, while others seem to do more harm than good. Through the analysis of specific bacterial roles in the GI tract and investigation of patterns of resistance and their associated side effects, an ideal of the "worst" antibiotic will be presented. Based on the largely inherent pathogenicity of gram-negative bacteria and the strong cascade of inflammatory effects they elicit, the "worst" antibiotic is one that is broad in its intended spectrum, yet it is associated with high levels of pathogenic, gram-negative resistance and low levels of commensal, gram-positive resistance. This type of resistance pattern is seen for Ceftriaxone, making it a very dangerous antibiotic in theory due to its resistance-dampened impact on pathogenic gram-negative species, yet maintained effectiveness on commensal, gram-positive species in the gut. Resistance patterns such as this allow for pathogen proliferation and increased intestinal permeability due to overgrowth into the normally commensal niches left behind by Ceftriaxone-susceptible organisms responsible for maintaining gut balance and intestinal integrity. Despite the limited power of causality provided by co-relational relationships, the high predictive power of Ceftriaxone use for hospital visits for sleep disorders, NEMIDs, and diabetes mellitus in comparison to narrow-spectrum Amoxicillin tends to support the mechanistic outputs of taking broad-spectrum agents with the aforementioned resistance patterns. By comparison, the most "ideal" antibiotic in theory would be one narrow in spectrum, demonstrating a very targeted mechanism of action, and eliciting 
little to no collateral damage on the endogenous species of the gut. If bacteria were to develop resistance from overuse of this antibiotic, then ideally it would be gram-positive bacteria, since a majority of the commensal microbes in the GI tract are gram-positive and are responsible for maintaining intestinal integrity, thus muting the niche clearance-associated overgrowth seen with $3 \mathrm{gc}$ use.

The proposition of the most "ideal" antibiotic theoretically promotes an increased use of narrow-spectrum antibiotics in a prescriber setting. However, this ideology reaches commercial roadblocks when considered in a real world context. Pharmaceutical companies are the main avenue of antibiotic distribution, yet they receive very low returns on new antibiotic production and exploration. These low returns are primarily based on the short-term use of antibiotics, with buyers experiencing an almost immediate termination of infection, thus discontinuing product use. From a producer point of view, this is not a profitable situation, as the consumer is no longer paying the pharmaceutical company once treatment is stopped. Additionally, the money needed to keep up with the rate of microbial resistance is not equally met in consumer purchases due to the inconsistency of infection. In contrast, pharmaceutical companies receive a significantly greater return on chronic illness medications, which a patient typically has to take for the rest of their life, thereby paying the pharmaceutical company for the rest of their life. Through this realization of profit maximization, pharmaceutical companies turn their drug development targets to these chronically ill "profitable humans", roughly marking human health as an undesirable commodity. As a result, pharmaceutical companies tend to offer a limited number of broad-spectrum antibiotics rather than a wide range of narrow-spectrum antibiotics as deemed ideal. Additionally, these broad-spectrum antibiotics are widely used and often improperly prescribed, contributing to an associated high level of resistance that promotes pathogenic overgrowth and commensal depletion [143]. At his Nobel Lecture in 1945, Fleming questioned who was responsible for death from an infectious disease, the microbe, or the individual who conditioned its resistance through improper use of the antibiotic [144].

The current economic and resistance ridden state of antibiotics, tends to suggest the modern age of antibiotics is coming to an end, much like the crisis associated with the depletion of fossil fuels, forcing a turn towards new resources. Adoption this view of antibiotics as a limited commodity, an increased responsibility can be assigned to the appropriateness of their use and an increased attention to the consideration of spectrum. However, based on the results and discussion presented in this study, perhaps the result lies within our microbes. By maintaining a balanced microbial ecology, especially in regard to commensal species, the need for antibiotic therapy in the first place may be avoided, as a balanced gut maintains diversity and prevents the opportunistic overgrowth commonly characterizing infections.

\section{Combined Antifungal and Antibiotic Therapy.}

The idea of getting an antifungal prescription with an antibiotic to treat a bacterial infection seems perplexing in its nature. However, this idea of dual microbial therapy is not a new one, as a 1976 article encourages the clinical effectiveness of its implementation [146]. This prescribing strategy could prove to be enormously beneficial in stopping antibiotic-induced infections, such as Candidiasis. As previously discussed, the symptoms manifesting from Candida overgrowth can be somewhat misleading and are often treated symptomatically due to the unknown etiology of the symptoms. However, through the prescription of an antifungal with the antibiotic, the opportunistic Candida species can be kept from expanding into these antibiotic-vacated niches, which prevents it from becoming pathogenic.

Recent pharmaceutical developments show promise in the future of this dual therapy as a new wave of "antifungal antibiotics" are being developed at an increased level [147, 148]. The ideal of these developments is one to be applauded, as the understanding of the diverse community harbored in the gut and the consequential effects antibiotic therapy in isolation can have on its symbiosis is being realized.

\section{Dietary approaches to maintain gut health Probiotics}

Antibiotics are very beneficial, as they function to wipe out disease-causing bacteria. However, depending on the spectrum and resistance patterns of the antibiotic, the undesirable effect of wiping out beneficial bacteria can also occur. It is starting to be understood that the reintroduction of commensal bacteria to the gut following the course of an antibiotic is of critical importance, which can be accomplished through probiotic supplements. Probiotics are live strains of commensal bacteria and yeast that support digestive function and intestinal integrity. Additionally, they critically serve to repopulate the niches vacated by antibiotic-susceptible organisms, which helps prevent opportunistic pathogen overgrowth [149]. The effectiveness of restoring commensal species is demonstrated in multiple studies, showing that downing a "Clostridial cocktail" can restore immune equilibrium and eliminate gut-mediated inflammatory responses [150, 151]. Another study noted the effectiveness of probiotic rather than antifungal use in treating Candida, as the reinstatement of acid-producing species found in probiotics restore an acidic intestinal pH that prevents Candida growth that was previously allowed due to the alkaline pH promoted by use of the antibiotic [152]. Studies like these should reinstate hope in our microbial symbionts, and raise personal awareness to how important the often-unseen function of these microbes are for our well being. Treat them inconsiderately and the negative consequences will manifest. However, when investment is placed in our microbial assets, the immediate returns can put even the pharmaceutical companies to shame.

\section{Concluding remarks}

\section{Going forward}

The correlations found in this study, along with their associated implications, suggests that broad-spectrum antibiotic use is causing a wide range of chronic side effects not previously connected to their use, and even suggest that they are losing their effectiveness in the treatment of infectious and parasitic disease 
(Figure 4A-D). Interpretation of the results and current experimental evidence encourages a paradigm shift from the view that all microbes are "bad", but rather that there exists an entire world of "good", commensal bacteria that have developed a symbiotic relationship with humans over time. This perspective should appropriately be accompanied by a promotion of behavior supporting the growth of commensal species and an avoidance of behavior that can wipe out these communities. One known way to wipe out these communities is through the use of broad-spectrum antibiotics, which can allow for the increase of "bad" bacteria to proliferate at the detriment of the "good". Evidence of this phenomenon is readily seen in opportunistic infections following the course of an antibiotic [111].

The concept that infectious disease is merely an uninhibited overgrowth of a microbial species, usually not present or kept in check, suggests a newfound openness to ensuring a maintenance of the commensal species that function to keep these pathogens in check. However, the idea of using bacteria to fight bacterial overgrowth is one that seems contradicting upon first read. Fortunately, a return to the Aristotelian ideology that virtue is found between the extremes of excess and depletion provides an understanding of how crucial the maintenance of microbial diversity is for existing in a virtuous state of health. Denying the incredible benefit antibiotics have provided to society would be asinine, however, a Hippocratic appraisal of their current state is warranted, prompting an increased faith in the endogenous symbionts inside of the gut. With this mindset, the need for novel antimicrobial therapy should be directed no further, as the promising answers to treatment of infectious and chronic disease lie right inside of the gut.

\section{References}

1 WHO. Physical status: the use and interpretation of anthropometry. Report of a WHO Expert Committee. WHO Technical Report Series 854. Geneva: World Health Organization. 1995.

2 Trust, America's Health, and Robert Wood Johnson Foundation. Obesity rates \& trends overview: The state of obesity. 2004.

3 WHO. Obesity and overweight. World Health Organization. 2016.

4 Kengne AP, Sobngwi E, Echouffo-Tcheugui JB, Mbanya JC. New Insights on Diabetes Mellitus and Obesity in Africa-Part 2: Prevention, Screening and Economic Burden. Heart. 2013;99(15):1072-1077. doi: 10.1136/heartjnl-2013-303773

5 Overweight \& Obesity. Adult obesity prevalence maps. CDC. 2016.

6 BRFFS. Behavioral Risk Factor Surveillance System Survey Data. CDC. 2015.

7 Ballard-Barbash R, Berrigan D, Potischman N, Dowling E. Obesity and cancer epidemiology. In: Berger NA, editor. Cancer and Energy Balance, Epidemiology and Overview. New York: Springer-Verlag New York, LLC, 2010.

8 Calabro S. The allergy and obesity link. Everyday Health com. 2009.

9 Devol R, Bedroussian A, Charuworn A, Chatterjee A, Kim I, Soojung K. An Unhealthy America: The Economic Burden of Chronic Disease. The minority health \& health enquity. 2007.

10 Wang YC, Klim McPherson, Tim Marsh, Steven L Gortmaker, Martin Brown. Health and Economic Burden of the Projected Obesity Trends in the USA and the UK. The Lancet. 2011;378(9793):815-825.

11 Polednak AP. Estimating the number of U.S. incident cancers attrib- utable to obesity and the impact on temporal trends in incidence rates for obesity-related cancers. Cancer Detection and Prevention. 2008;32(3):190-199. doi: 10.1016/j.cdp.2008.08.004

12 a. CDC. Achievements in public health, 1900-1999: Changes in the public health system. MMWR. 1999;48(50):1141-1147

12 b. Collins, Selwyn D. Excess Mortality from Causes Other Than Influenza and Pneumonia during Influenza Epidemics. Public Health Reports (1896-1970). 1932;47(46):2159-2179.

13 Gould IM, Bal AM. New antibiotic agents in the pipeline and how they can overcome microbial resistance. Virulence.2013;4(2):185-191. doi: $10.4161 /$ viru.22507

14 Spellberg B, Gilbert DN. The future of antibiotics and resistance: a tribute to a career of leadership by John Bartlett. Clin Infect Dis. 2014;59(suppl 2):S71-S75. doi: 10.1093/cid/ciu392

15 CDC. Antibiotic / Antimicrobial resistance. CDC. 2016.

16 Slonczewski, Joan L, John W Foster, and Kathy M Gillen. Microbiology: An Evolving Science (Second Edition). 2nd ed. New York: Norton, W. W. \& Company, 2011. Print.

17 Spectrum of activity - Antimicrobial resistance learning site for veterinary students. 2011. Web. 2016.

18 Sarpong EM, Miller GE. Narrow- and Broad-Spectrum Antibiotic Use among U.S. Children. Health Serv Res. 2015;50(3):830-846. doi: 10.1111/1475-6773.12260

19 CDC. Measuring outpatient antibiotic prescribing. CDC, 21 Apr. 2016.

20 Mc Caig LF, Burt CW. National Hospital Ambulatory Medical Care Survey: 2001 Emergency Department Summary. Hyattsville, Mary- land: National Center for Health Statistics; 2003. Advance Data from Vital and Health Statistics. 2003;(335):1-29.

21. Food and Drug Administration. National Drug Code Directory, 1995 Edition. Washington, D.C. Public Health Service. 1995.

22. Lee GC, Reveles KR, Attridge RT, Lawson KA, Mansi IA, Lewis JS 2nd, et al. Outpatient Antibiotic Prescribing in the United States: 2000 to 2010. BMC Med. 2014;12:96. doi:10.1186/1741-7015-12-96

23. Murphy Ryan Anne. Prevalence of Inappropriate Antibiotic Prescriptions Among US Ambulatory Care Visits, 2010-2011. The Journal of Emergency Medicine. 2016;51(3):337.

24. Sengupta S, Chattopadhyay MK, Grossart HP. The multifaceted roles of antibiotics and antibiotic resistance in nature. Front Microbiol. 2013;4:47. doi:10.3389/fmicb.2013.00047

25. Qin J, Li R, Raes J, Arumugam M, Burgdorf KS, Manichanh C, et al. A human gut microbial gene catalogue established by metagenomic sequencing. Nature. 2010;464(7285): 59-65. doi:10.1038/nature08821

26. Ursell LK, Metcalf JL, Parfrey LW, Knight R. Defining the Human Microbiome. Nutr Rev. 2012;70(Suppl 1):S38-S44. doi:10.1111/j.17534887.2012.00493.x

27. Turnbaugh PJ, Ley RE, Hamady M, Liggett CF, Knight R, Gordon JI. The Human Microbiome Project: Exploring the Microbial Part of Ourselves in a Changing World. Nature. 2007;449(7164):804-810. doi:10.1038/ nature 06244

28. Penton, Leidon. Probiotics may keep you from Dwelling on the past. New Hope Network. 2016.

29. Kostic AD, Gevers D, Siljander H, Vatanen T, Hyötyläinen T, Hämäläinen AM, et al. The Dynamics of the Human Infant Gut Microbiome in Development and in Progression towards Type 1 Diabetes. Cell Host Microbe. 2015;17(2):260-273. doi:10.1016/j.chom.2015.01.001

30. Hullar MA, Fu BC. Diet, the Gut Microbiome, and epigenetics. Cancer J. 2014;20(3):170-175. doi: 10.1097/PP0.0000000000000053

31. De Angelis M, Piccolo M, Vannini L, Siragusa S, De Giacomo A, Serrazzanetti DI, et al. Fecal Microbiota and Metabolome of Children with Au- 
tism and Pervasive Developmental Disorder Not Otherwise Specified. PLoS One. 2013;8(10):e76993. doi:10.1371/journal.pone.0076993

32. Mangiola F, Ianiro G, Franceschi F, Fagiuoli S, Gasbarrini G, Gasbarrini A. Gut Microbiota in Autism and Mood Disorders. World J Gastroenterol. 2016;22(1):361-368. doi:10.3748/wjg.v22.i1.361

33. Paget SA. The Microbiome, Autoimmunity, and Arthritis: Cause and Effect: An Historical Perspective. Trans Am Clin Climatol Assoc. 2012;123:257-267.

34. Aitken JD, Gewirtz AT. Gut Microbiota in 2012: Toward Understanding and Manipulating the Gut Microbiota. Nat Rev Gastroenterol Hepatol. 2013;10(2):72-74. doi:10.1038/nrgastro.2012.252

35. Hooper LV, Gordon JI. Commensal Host-Bacterial Relationships in the Gut. Science. 2001;292(5519):1115-1118.

36. D'Argenio V, Salvatore F. The Role of the Gut Microbiome in the Healthy Adult Status. Clinica Chimica Acta. 2015;451:97-102.

37. a. Flint HJ, Scott KP, Duncan SH, Louis P, Forano E. Microbial Degradation of Complex Carbohydrates in the Gut. Gut Microbes. 2012;3(4):289-306. doi:10.4161/gmic.19897

37 b. Rothe M, Blaut M. Evolution of the Gut Microbiota and the Influence of Diet. Benef Microbes. 2013;4(1):31-37. doi: 10.3920/BM2012.0029

38 Spronk HM, Soute BA, Schurgers LJ, Thijssen HH, De Mey JG, Vermeer C. Tissue-Specific Utilization of Menaquinone-4 Results in the Prevention of Arterial Calcification in Warfarin-Treated Rats. J Vasc Res. 2003;40(6):531-537.

39 Eletriby A, Gomaa Y, Elmashhady Y. Coronary Calcium Score as an Independent Predictor of Adverse Events on Percutaneous Coronary Intervention Outcome. Ain Shams Medical Journal. 2013;64(4-6):327331. doi: $10.12816 / 0013875$

40 Betts, Gordon J. Anatomy \& Physiology. 2013. Print.

41 Goudswaard J. Mucosal Immune System. Veterinary Immunology and Immunopathology. 1983;4(4):513-514.

42 Tanaka S, Kobayashi T, Songjinda P, Tateyama A, Tsubouchi M, Kiyoha$\mathrm{ra} \mathrm{C}$, et al. Influence of antibiotic exposure in the early postnatal period on the development of intestinal microbiota. FEMS Immunol Med Microbiol. 2009;56(1):80-87. doi:10.1111/j.1574-695X.2009.00553.x

43 Dharmsathaphorn K. Regulation of tight junction permeability in the intestinal epithelium. Gastroenterology. 1989;97(3):802-803.

44 Abbas, Abul K, Andrew H Lichtman, and David L. Baker. Basic Immunology: Functions and Disorders of the Immune System [With Access Code] - 3rd Edition. 3rd ed. Philadelphia, PA: Elsevier Health Sciences. 2010.

45 Mantis NJ, Rol N, Corthésy B. Secretory IgA's complex roles in immunity and mucosal homeostasis in the gut. Mucosal Immunol. 2011;4(6):603-611. doi:10.1038/mi.2011.41

46 Williams, Andrew E, and Tracy Hussell. Immunity: Mucosal Immunology in Health and Disease. United States: John Wiley \& Sons. 2011.

47 Tlaskalová-Hogenová H, Tučková L, Lodinová-Žádniková R, Štěpánková R, Cukrowska B, Funda DP, et al. Mucosal immunity: Its role in defense and allergy. Int Arch Allergy Immunol. 2002;128(2):77-89.

48 Liu Z, Li N, Neu J. Tight junctions, leaky intestines, and pediatric diseases. Acta Paediatr. 2005;94(4):386-393.

49 Jialal I, Rajamani U. Endotoxemia of Metabolic Syndrome: A Pivotal Mediator of Meta-Inflammation. Metab Syndr Relat Disord. 2014;12(9):454-456. doi:10.1089/met.2014.1504

50 Apovian C. A high-fat meal induces low-grade endotoxemia: Evidence of a novel mechanism of postprandial inflammation. Yearbook of Endocrinology. 2008;120-121.

51 Neves AL, Coelho J, Couto L, Leite-Moreira A, Roncon-Albuquerque R Jr. Metabolic endotoxemia: A molecular link between obesity and car- diovascular risk. J Mol Endocrinol. 2013;51(2):R51-64. doi: 10.1530/ JME-13-0079

52 Arrieta MC, Bistritz L, Meddings JB. Alterations in intestinal permeability. Gut. 2006;55(10):1512-1520. doi: 10.1136/gut.2005.085373

53 Velasquez OR, Tso P, Crissinger KD. Fatty acid-induced injury in developing piglet intestine: Effect of degree of saturation and carbon chain length. Pediatr Res. 1993;33(6):543-547.

54 Devaraj S, Hemarajata P, Versalovic J. The Human Gut Microbiome and Body Metabolism: Implications for Obesity and Diabetes. Clin Chem. 2013;59(4):617-628. doi: 10.1373/clinchem.2012.187617

55 Ley RE, Turnbaugh PJ, Klein S, Gordon JI. Microbial Ecology: Human Gut Microbes Associated with Obesity. Nature. 2006;444(7122):10221023. doi: $10.1038 / 4441022 a$

56 Cox LM, Blaser MJ. Antibiotics in Early Life and Obesity. Nat Rev Endocrinol. 2015;11(3):182-190. doi: 10.1038/nrendo.2014.210.

57 Peter J Turnbaugh, Micah Hamady, Tanya Yatsunenko, Jeffrey I Gordon. A Core Gut microbiome in Obese and Lean Twins. Nature. 2008;457(7228):480-484.

58 Ridaura VK, Faith JJ, Rey FE, Cheng J, Duncan AE, Kau AL, et al. Gut Microbiota from Twins Discordant for Obesity Modulate Metabolism in Mice. Science. 2013;341(6150):1241214. doi: 10.1126/science.1241214

59 Williams, David. Gut flora, losing weight, and the real reason gastric bypass works. David Williams. 2016.

60 Round JL, Mazmanian SK. The Gut Microbiota Shapes Intestinal Immune Responses During Health and Disease. Nat Rev Immunol. 2009;9(5):313-323. doi: 10.1038/nri2515

61 Ley RE, Turnbaugh PJ, Klein S, Gordon JI. Microbial Ecology: Human Gut Microbes Associated with Obesity. Nature. 2006;444(7122):10221023. doi: $10.1038 / 4441022 a$

62 Bessesen DH. An Obesity-Associated Gut Microbiome with Increased Capacity for Energy Harvest. Yearbook of Endocrinology. 2007;163165.

63 Fei Na, and Liping Zhao. An Opportunistic Pathogen Isolated from the Gut of an Obese Human Causes Obesity in Germfree Mice. The ISME Journal. 2012;7(4):880-884. doi: 10.1038/ismej.2012.153

64 Sezenna M. Proteobacteria: Phylogeny, metabolic diversity and ecological effects. 2004.

65 Narushima S, Hase K, Kim S, Fritz JV, Wilmes P, Ueha S, et al. Treg induction by a rationally selected mixture of Clostridia strains from the human microbiota. Nature. 2013;500(7461):232-236. doi: 10.1038/ nature12331

66 Dethlefsen L, Huse S, Sogin ML, Relman DA. The pervasive effects of an antibiotic on the human gut microbiota, as revealed by deep $16 \mathrm{~S}$ rRNA sequencing. PLoS Biol. 2008;6(11):e280. doi: 10.1371/journal. pbio.0060280

67 Fleissner CK, Huebel N, Abd El-Bary MM, Loh G, Klaus S, Blaut M. Absence of intestinal microbiota does not protect mice from dietinduced obesity. Br J Nutr. 2010;104(6):919-929. doi: 10.1017/ S0007114510001303

68 Dethlefsen L, Huse S, Sogin ML, Relman DA. The Pervasive Effects of an Antibiotic on the Human Gut Microbiota, as Revealed by Deep 16S rRNA Sequencing. Jonathan A Eisen. PLoS Biol. 2008;6(11):e280. doi: 10.1371/journal.pbio.0060280

69 Pearson, H. Fat people harbour fat microbes. Nature. 2006. doi:10.1038/news061218-6

70 Pearson, H. Stomach bug makes food yield more calories. Nature. 2006. doi:10.1038/news060522-19

71 Lawrence J. Brandt. Fecal Transplantation for the Treatment 
of Clostridium Difficile Infection. Gastroenterol Hepatol (N Y). 2012;8(3):191-194.

72 Gens KD, RH Elshaboury, JS Holt. Fecal Microbiota Transplantation and Emerging Treatments for Clostridium Difficile Infection. J Pharm Pract. 2013;26(5):498-505. doi: 10.1177/0897190013499527

73 Ambrose NS, Johnson M, Burdon DW, Keighley MR. The Influence of Single Dose Intravenous Antibiotics on Faecal Flora and Emergence of Clostridium Difficile. J Antimicrob Chemother. 1985;15(3):319-326.

74 Dethlefsen L, Huse S, Sogin ML, Relman DA. The Pervasive Effects of an Antibiotic on the Human Gut Microbiota, as Revealed by Deep 16S RRNA Sequencing. PLoS Biol. 2008;6(11):e280. doi: 10.1371/journal. pbio.0060280

75 Morgun A, Dzutsev A, Dong X, Greer RL, Sexton DJ, Ravel J, et al. Uncovering Effects of Antibiotics on the Host and Microbiota Using Transkingdom Gene Networks. Gut. 2015;64(11):1732-1743. doi: 10.1136/ gutjnl-2014-308820

76 Jakobsson HE, Jernberg C, Andersson AF, Sjolund-Karlsson M, Jansson JK, Engstrand L. Short-term antibiotic treatment has differing longterm impacts on the human throat and gut microbiome. PLoS One. 2010;5(3):e9836. doi: 10.1371/journal.pone.0009836

77 Tap J, Furet JP, Bensaada M, Philippe C, Roth H, Rabot S, et al. Gut Microbiota Richness Promotes Its Stability Upon Increased Dietary Fibre Intake in Healthy Adults. Environ Microbiol. 2015;17(12):4954-4964. doi: 10.1111/1462-2920.13006

78 Le Chatelier E, Nielsen T, Qin J, Prifti E, Hildebrand F, Falony G, et al. Richness of Human Gut Microbiome Correlates with Metabolic Markers. Nature. 2013;500(7464):541-546. doi: 10.1038/nature12506

79 Brüssow H. Growth Promotion and Gut Microbiota: Insights from Antibiotic Use. Environ Microbiol. 2015;17(7):2216-2227. doi: 10.1111/1462-2920.12786

80 Ng KM, Ferreyra JA, Higginbottom SK, Lynch JB, Kashyap PC, Gopinath $\mathrm{S}$, et al. Microbiota-Liberated Host Sugars Facilitate Post-Antibiotic Expansion of Enteric Pathogens. Nature. 2013;502(7469):96-99. doi: $10.1038 /$ nature 12503

81 Hall IC, O'Toole E. Intestinal flora in new-born infants with a description of a new pathogenic anaerobe, Bacillus difficilis. Am J Dis Child. 1935;49:390-402. doi:10.1001/archpedi.1935.01970020105010

82 Rea MC, Dobson A, O’Sullivan O, Crispie F, Fouhy F, Cotter PD, et al. Effect of Broad- and Narrow-Spectrum Antimicrobials on Clostridium Difficile and Microbial Diversity in a Model of the Distal Colon. Proc Natl Acad Sci USA. 2011;108 (Suppl 1):4639-4644. doi: 10.1073/ pnas.1001224107.

83 Centers for Disease Control and Prevention (CDC). Behavioral Risk Factor Surveillance System Survey Data. Atlanta, Georgia: U.S. Department of Health and Human Services, Centers for Disease Control and Prevention, 1993-2008. 2011-2014.

84 Merrill RM, Richardson JS. Validity of self-reported height, weight, and Body Mass Index: Findings from the National Health and Nutrition Examination Survey, 2001-2006. Prev Chronic Dis. 2009;6(4):A121.

85 NAMCS description: US Centers for Disease Control and Prevention. Available from: http://www.cdc.gov/nchs/about/major/ahcd/namcsdes.htm

86 Mainous AG III, Hueston WJ, Davis MP, Pearson WS. Trends in antimicrobial prescribing for bronchitis and upper respiratory infections among adults and children. Am J Public Health. 2003;93(11):19101914.

87 NAMCS/NHAMCS - ambulatory care drug database system - search. 2016. Available from: https://www2.cdc.gov/drugs/applicationnav1. asp
88 Boardman C. System and method for estimating prdouct distribution using a product specific universe US 7174304 B1. IMS Health Incorporated. 2007.

89 Hicks LA, Bartoces MG, Roberts RM, Suda KJ, Hunkler RJ, Taylor TH, et al. US Outpatient Antibiotic Prescribing Variation According to Geography, Patient Population, and Provider Specialty in 2011. Clin Infect Dis. 2015;60(9):1308-1316.

90 The Uniform System of Classification (USC). IMS Health. 2016. Available from: https://www.imshealth.com/files/web/IMSH\%20Institute/USC_Classiification_Process_2011.pdf

91 Kohanski MA, Dwyer DJ, Collins JJ. How Antibiotics Kill Bacteria: From Targets to Networks. Nat Rev Microbiol. 2010;8(6):423-435. doi: 10.1038/nrmicro2333

92 Monika IK. Molecular Targets of $\beta$-Lactam-Based Antimicrobials: Beyond the Usual Suspects. Antibiotics (Basel). 2014;3(2):128-142.

93 Mainous AG III, Hueston WJ, Davis MP, Pearson WS. Trends in antimicrobial prescribing for bronchitis and upper respiratory infections among adults and children. Am J Public Health. 2003;93(11):19101914.

94 Rafii F, Sutherland JB, Cerniglia CE. Effects of Treatment with Antimicrobial Agents on the Human Colonic Microflora. Ther Clin Risk Manag. 2008;4(6):1343-1358.

95 Giuliano M, Barza M, Jacobus NV, Gorbach SL. Effect of Broad-Spectrum Parenteral Antibiotics on Composition of Intestinal Microflora of Humans. Antimicrob Agents Chemother. 1987;31(2):202-206.

96 Rea MC, Dobson A, O'Sullivan O, Crispie F, Fouhy F, Cotter PD, et al. Effect of Broad- and Narrow-Spectrum Antimicrobials on Clostridium Difficile and Microbial Diversity in a Model of the Distal Colon. Proc Natl Acad Sci USA. 2011;108(Suppl 1):4639-4644. doi: 10.1073/ pnas. 1001224107

97 National Hospital Ambulatory Medical Care Survey: Emergency Department Summary [NHSR No. 7]. 2008. Available from: https://www. cdc.gov/nchs/data/nhsr/nhsr007.pdf

98 Andes DR, Craig WA. Cephalosporins. In: Mandell GL, Bennett JE, Dolin R, Eds. Principles and Practice of Infectious Diseases. 7th ed. Philadelphia, PA: Churchill, Livngstone. Elsevier. 2009.

99 Gilbert MD, David N. Sanford Guide to Antimicrobial Therapy 2014. United States: Antimicrobial Therapy. 2014.

100 Fuchs PC, Barry AL, Pfaller MA, Hardy DJ, McLaughlin JC, Gerlach EH. In Vitro Activity of Ampicillin, Amoxicillin, AmpicillinSulbactam, and Amoxicillin-Clavulanic Acid Against Consecutive Clinical Isolates of Enterobacteriaceae. Diagn Microbiol Infect Dis. 1993;17(2):171-175.

101Blommaert A, Coenen S, Gielen B, Goossens H, Hens N, Beutels P. Patient and Prescriber Determinants for the Choice Between Amoxicillin and Broader-Spectrum Antibiotics: A Nationwide PrescriptionLevel Analysis. J Antimicrob Chemother. 2013;68(10):2383-2392. doi: 10.1093/jac/dkt170

102Le Chatelier E1, Nielsen T, Qin J, Prifti E, Hildebrand F, Falony G, et al. Richness of human gut microbiome correlates with metabolic markers. Nature. 2013;500(7464):541-546. doi: 10.1038/nature12506.

103Fang S, Evans RM. Microbiology: Wealth Management in the Gut. Nature. 2013;500(7464):538-539. doi: 10.1038/500538a

104.Cotillard A, Kennedy SP, Kong LC, Prifti E, Pons N, Chatelier EL, et al. Corrigendum: Dietary Intervention Impact on Gut Microbial Gene Richness. Nature. 2013;500(7464):585-588. doi: 10.1038/nature 12480

105.Bundey S. International Nomenclature of Diseases. Vol VI. Metabolic, Nutritional and Endocrine Disorders. J Med Genet. 1992;29(8):599- 
600.

106.Blumstein DT, Levy K, Mayer E, Harte J. Gastrointestinal Dysbiosis. Evol Med Public Health. 2014:163-163.

107.Johnson AR, Milner JJ, Makowski L. The Inflammation Highway: Metabolism Accelerates Inflammatory Traffic in Obesity. Immunol Rev. 2012;249(1):218-238. doi: 10.1111/j.1600-065X.2012.01151.x

108.Harte J. Maximum Entropy and Ecology: A Theory of Abundance, Distribution, and Energetics. Oxford, UK: Oxford University Press. 2011.

109.Fielding, Henry, et al. Aristotle: Nicomachean Ethics, Books Ii--Iv. New York: Oxford University Press. 1998. Print.

110.Kamada N, Chen GY, Inohara N, Núñez G. Control of pathogens and pathobionts by the gut microbiota. Nat Immunol. 2013;14(7):685690. doi: $10.1038 /$ ni.2608

111.Dancer SJ. The Problem with Cephalosporins. Journal of Antimicrobial Chemotherapy. 2001;48(4):463-478.

112.Ruppe E, Woerther PL, Barbier F. Mechanisms of antimicrobial resistance in Gram-negative bacilli. Ann Intensive Care. 2015;5(1):21. doi: 10.1186/s13613-015-0061-0

113.Klein NC, Cunha BA. Third Generation Cephalosporins. Med Clin North Am. 199;79(4):705-719.

114.Wise, R. $\beta$-Lactams: cephalosporins. In Antibiotics and Chemotherapy, 7 th edn, (O'Grady, F., Lambert, P. H., Finch, R. G., Greenwood, D., Eds), pp. 202-55. Churchill Livingstone, New York.

115 Pallares R, Pujol M, Pena C, Ariza J, Martin F \& Gudiol F. Cephalosporins as risk factors for nosocomial Enterococcus faecalis bacteraemia. A matched case-control study. Archives of Internal Medicine. 1993;153(13):1581-1586.

116 Moellering RC. Emergence of Enterococcus as a significant pathogen. Clinical Infectious Diseases. 1992;14(6):1173-1176.

117 Girbes Armand R J, René Robert, Paul E Marik. The Dose Makes the Poison. Intensive Care Medicine. 2016;42(4):632-632.

118 Guggenbichler JP, Allerberger FJ \& Dierich M. Influence of cephalosporins III generation with varying biliary excretion on faecal flora and emergence of resistant bacteria during and after cessation of therapy. Padiatrie und Padologie. 1986;21(4):335-342.

119 Kinsman OS, Pitblado K. Candida albicans gastrointestinal colonization and invasion in the mouse: effect of antibacterial dosing, antifungal therapy and immunosuppression. Mycoses. 1989;32(12):664-674.

120 Samonis G, Dassiou M, Anastassiadou H. Antibiotics affecting gastrointestinal colonization of mice by yeasts. Journal of Chemotherapy. 1994;6(1):50-52.

121 Magnussen CR, Cave J. Nosocomial enterococcal infections: association with use of third-generation cephalosporin antibiotics. American Journal of Infection Control. 1988;16(6):241-245.

122 Leigh DA, Walsh B, Leung A, Tait S, Peatey K, Hancock P. The effect of cefuroxime axetil on the faecal flora of healthy volunteers. Journal of Antimicrobial Chemotherapy. 1990;26(2):261-268.

123 Edlund C, Nord CE. A model of bacterial- antimicrobial interactions: the case of oropharyngeal and gastrointestinal microflora. Journal of Chemotherapy. 1991;(3 Suppl 1):196-200.

124 Rao Jaladanki N, Jian-Ying Wang. Regulation of Gastrointestinal Mucosal Growth. San Rafael, CA: Morgan \& Claypool Life Sciences. 2010.

125 WR Wikoff, AT Anfora, J Liu, PG Schultz, SA Lesley, EC Peters, et al. Metabolomics analysis reveals large effects of gut microflora on mammalian blood metabolites. Proc. Natl. Acad. Sci. USA. 2009;106(10):36983703. doi: $10.1073 /$ pnas. 0812874106

126 Venkatesh Madhukumar, Mukherjee S, Wang H, Li H, Sun K, Benechet AP, et al. Symbiotic Bacterial Metabolites Regulate Gastrointestinal Barrier Function via the Xenobiotic Sensor PXR and Toll-Like
Receptor 4. Immunity. 2014;21(4):296-310. doi: 10.1016/j.immuni.2014.06.014.

127 ML Hart, KA Ceonzo, LA Shaffer, K Takahashi, RP Rother, WR Reenstra, et al. Gastrointestinal ischemia-reperfusion Injury is lectin complement pathway dependent without involving C1q. J Immunol. 2005;174(10):6373-6380.

128 Magnussen CR, Cave J. Nosocomial enterococcal infections: association with use of third-generation cephalosporin antibiotics. American Journal of Infection Control. 1988;16(6):241-245.

129 Kitchens RL, Ulevitch RJ, Munford RS. Lipopolysaccharide (LPS) Partial Structures Inhibit Responses to LPS in a Human Macrophage Cell Line Without Inhibiting LPS Uptake by a CD14- Mediated Pathway. Journal of Experimental Medicine. 1992;176(2):485-494.

130 Jialal Ishwarlal, Uthra Rajamani. Endotoxemia of Metabolic Syndrome: A Pivotal Mediator of Meta-Inflammation. Metabolic Syndrome and Related Disorders. 2014;12(9):454-456. doi: 10.1089/ met.2014.1504.

131 Nilsson C, Larsson BM, Jennische E, Eriksson E, Björntorp P, York DA, et al. Maternal Endotoxemia Results in Obesity and Insulin Resistance in Adult Male Offspring. Endocrinology. 2001;142(6):2622-2630.

132 Lang Charles H, Silvis C, Deshpande N, Nystrom G, Frost RA. Endotoxin Stimulates in Vivo Expression of Inflammatory Cytokines Tumor Necrosis Factor Alpha, Interleukin-1, -6, and High-Mobility-Group Protein-1 in Skeletal Muscle. Shock. 2003;19(6):538-546.

133 Wang Zhaoxia, Tomohiro Nakayama. Inflammation, a Link Between Obesity and Cardiovascular Disease. Mediators of Inflammation. 2010;(2010):1-17. doi: 10.1155/2010/535918.

134 Bosma-den Boer Margarethe M, Marie-Louise van Wetten and Leo Pruimboom. Chronic Inflammatory Diseases Are Stimulated by Current Lifestyle: How Diet, Stress Levels and Medication Prevent Our Body from Recovering. Nutrition \& Metabolism. 2012;9(1):32. doi: 10.1186/1743-7075-9-32.

135 A Kubaszek, Pihlajamäki J, Komarovski V, Lindi V, Lindström J, Eriksson J, et al. Promoter polymorphisms of the TNF- $\alpha$ (G-308A) and IL-6 $(\mathrm{C}-174 \mathrm{G})$ genes predict the conversion from impaired glucose tolerance to type 2 diabetes: the Finnish diabetes prevention study. Diabetes. 2003;52(7):1872-1876.

136 Baker, Harriet. Homology as a Tool in Neuroscience: 2nd Annual Karger Workshop, St. Louis, Mo., October 1990. Special Topic Issue: 1991;38:4-5. Brain, Behavior and Evolution. Ed. H. Baker. Basel, Switzerland: S Karger AG. 1991. Print.

137 Ionescu Dawn F, Niciu Mj, Mathews Dc, Richards Em, Zarate Ca Jr. Neurobiology of anxious depression: a review. Depression and Anxiety. 2013;30(4):374-385. doi: 10.1002/da.22095

138 Elmenhorst David, Kroll T, Matusch A, Bauer A. Sleep Deprivation Increases Cerebral Serotonin 2A Receptor Binding in Humans. Sleep. 2012;35(12):1615-1623. doi: 10.5665/sleep.2230.

139 Musselman Dominique L, Charles B Nemeroff. The Role of Corticotropin-Releasing Factor in the Pathophysiology of Psychiatric Disorders. Psychiatric Annals. 1993;23(12):676-681.

140 Das Undurti. Metabolic Syndrome X Is a Low-Grade Systemic Inflammatory Condition with Its Origins in the Perinatal Period. Current Nutrition \& Food Science. 2007;3(4): 277-295.

141 Lotrich Francis. Inflammatory Cytokines, Growth Factors, and Depression. Current Pharmaceutical Design. 2012;18(36):5920-5935.

142 Hotamisligil GS, Shargill NS, Spiegelman BM. Adipose expression of tumor necrosis factor- $\alpha$ : direct role in obesity-linked insulin resistance. Science. 1993;259(5091):87-91.

143 Gonzales R, Barrett PH, Crane LA, John F. Steiner. Factors Associ- 
ated with Antibiotic Use for Acute Bronchitis. J Gen Intern Med. 1998;13(8):541-548. doi:10.1046/j.1525-1497.1998.00165.

144 Fleming, Sir Alexander. Penicillin. The American Journal of the Medical Sciences 1947; 214(2):224.

145 WHO. Race Against Time to Develop New Antibiotics. Bulletin of the World Health Organization. 2011;89(2):88-89.

146 Kozinn PJ, CL Taschdjian. Combined Antifungal and Antibiotic Therapy. BMJ . 1967;3(5560):307-308.

147 Oki Toshikazu, Konishi M, Tomatsu K, Tomita K, Saitoh K, Tsunakawa M, et al. Pradimicin, a Novel Class of Potent Antifungal Antibiotics. The Journal of Antibiotics. 1988;41(11):1701-1704

148 Evans David A, Brian T. Connell. Synthesis of the Antifungal Macrolide Antibiotic (+)-Roxaticin (I). J Am Chem Soc. 2003;125(36):1089910905.
149. Ritchie Marina L, Tamara N Romanuk. A Meta-Analysis of Probiotic Efficacy for Gastrointestinal Diseases. PLoS ONE . 2012;7(4):e34938. doi: 10.1371/journal.pone.0034938.

150 Atarashi K, Tanoue T, Oshima K, Suda W, Nagano Y, Nishikawa H, et al. Treg induction by a rationally selected mixture of Clostridia strains from the human microbiota. Nature. 2013;500(7461):232-236. doi: $10.1038 /$ nature 12331

151 Russell SL, Gold MJ, Willing BP, Thorson L, McNagny KM, Finlay BB. Perinatal antibiotic treatment affects murine microbiota, immune responses and allergic asthma. Gut Microbes. 2013;4(2):158-164. doi: 10.4161/gmic.23567.

152.De Oliveira Leite AM, Miguel MA, Peixoto RS, Rosado AS, Silva JT, Paschoalin VM. Microbiological, Technological and Therapeutic Properties of Kefir: A Natural Probiotic Beverage. Brazilian Journal of Microbiology. 2013;44(2):341-349. doi: 10.1590/S1517-83822013000200001 